UNITED STATES DEPARTMENT OF THE INTERIOR

GEOLOGICAL SURVEY

SCHLUMBERGER SOUNDINGS NEAR NEWBERRY CALDERA, OREGON

By

Robert J. Bisdorf

Open File Report $83-825$

1983

This report is preliminary and has not been reviewed for conformity with U.S. Geological Survey editorial standards. Any use of trade names is for descriptive purposes only and does not constitute endorsement by the USGS. 


\title{
Schlumberger Soundings near Newberry Caldera, Oregon
}

\author{
by
}

Robert J. Bisdorf

\section{INTRODUCTION}

In 1982 the U.S. Geologica1 Survey made 21 Schlumberger soundings in and near Newberry Caldera in Oregon (fig. 1). The soundings were made as part of the U.S.G.S.'s Geothermal program. The purpose of the soundings was to find areas of possible geothermal potential and to map zones of hot water which have been detected by drilling (Samme1, 1981).

Newberry Volcano, located about $40 \mathrm{~km}$ south of Bend, Oregon, is one of the largest Quaternary volcanoes in the conterminous United States. The volcano and its lava flows cover an area of greater than $1200 \mathrm{~km}^{2}$. Paulina Peak (elevation $2434 \mathrm{~m}$ ) is the highest remnant of the former mountain. The floor rocks and ejecta that form the volcano range in composition from basaltic to rhyolitic (Sammel, 1981).

The Newberry caldera (fig. 1) covers an area of about $45 \mathrm{~km}^{2}$. exposed rocks within the caldera are Quaternary in age. The present caldera floor is composed mainly of rhyolitic domes and flows of obsidian, basalt, and andesite (McLeod and others, 1981). Two lakes, Paulina and East, are located within the caldera. A line of hot springs occurs along the south shore of East Lake and a few springs occur on the north shore of Paulina Lake.

In 1981 the USGS drilled a geothermal test well in the caldera floor, to a depth of $930 \mathrm{~m}$. Water temperature at the bottom of the hole was $256^{\circ} \mathrm{C}$. The upper $610 \mathrm{~m}$ of the hole was described as very permeable and the lower 320 m as probably quite impermeable (Samme1, 1981). 


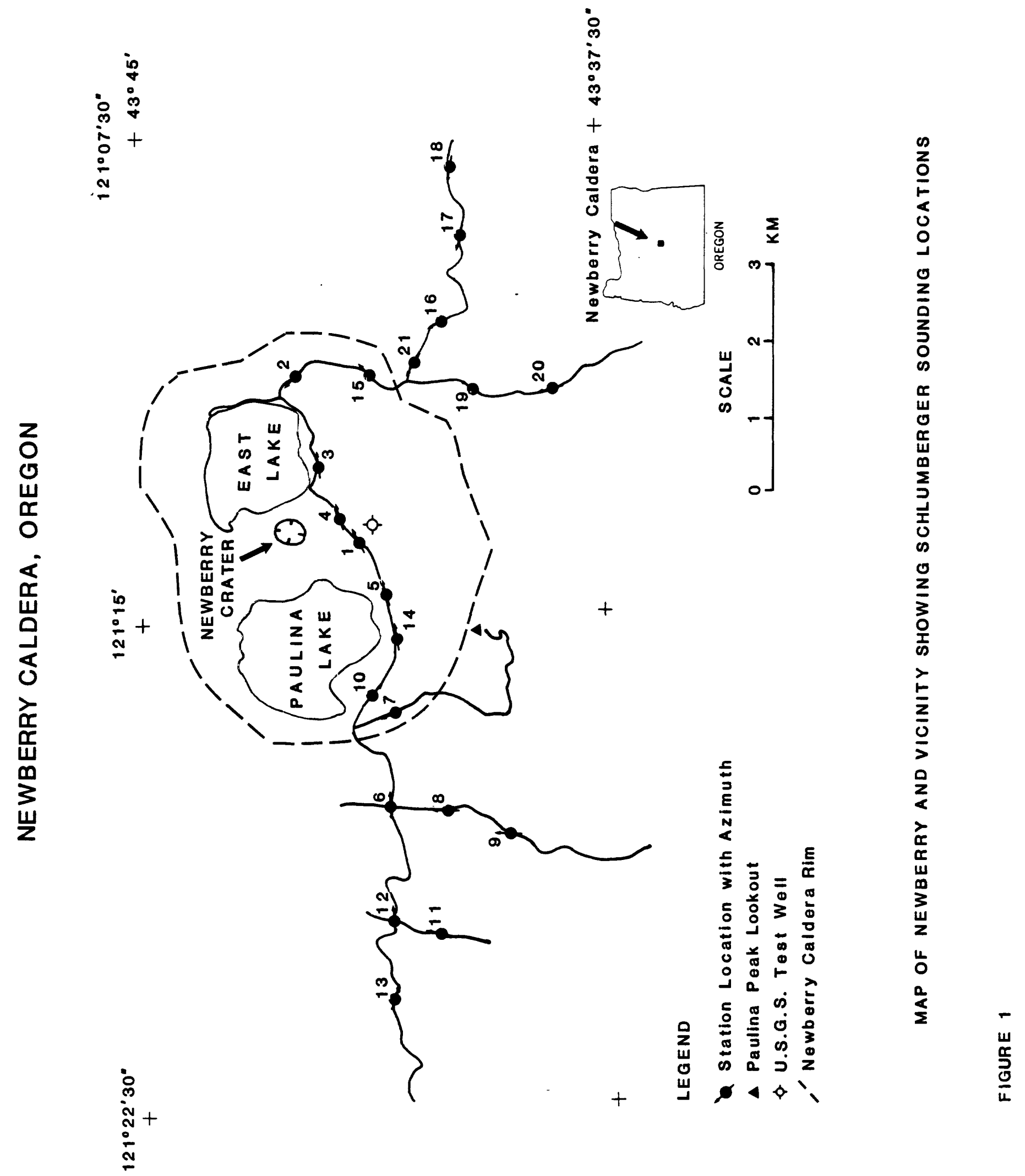


The purpose of this report is to release the Schlumberger sounding data. Automatic inversions and a geoelectric cross section are also given and discussed.

\section{SCHLUMBERGER SOUNDINGS}

Figure 1 is a map showing the location, identifying number, and direction of expansion of the Schlumberger soundings. The Schlumberger soundings are numbered consecutively from Newberry 1 to Newberry 21 . All the soundings were made along existing roads. Soundings were corrected for road curvature in a manner similar to that described by Zohdy and Bisdorf (1982). All the sounding data were automatically processed and interpreted (Zohdy, 1973; Zohdy, 1975) as shown in the graphs in the Appendix. The curves were interpreted on a Hewlett-Packard (HP) 9845B desk top computer using a program based on that of Zohdy (1973). The HP program was modified to use 0'Neil1 coefficients (0'Nei11, 1975) in place of Ghosh coefficients (Ghosh, 1971).

For each sounding, the data in the Appendix include:

1) A log-log plot of the field data points, in which the "O"'s represent. the individual data points. The $\mathrm{AB} / 2$ electrode spacings, which were measured in feet, have been converted to meters. Each set of data points that was made with the same potential electrode spacing (MN) is connected with a solid line. Measurements were made at the fixed $\mathrm{MN} / 2$ spacings of $2,6,20,60,200$, and 600 feet.

2) A tabulation of the $\mathrm{AB} / 2$ electrode spacings in meters and the corresponding apparent resistivities in ohm-m.

3) A log-log plot of the output of the automatic inversion program in which:

a) The continuous curve represents the shifted-digitized field curve (Bisdorf and Zohdy, 1979). 
b) The step-function curve represents the distribution of interpreted-true resistivity with depth.

c) The plus (+) signs represent points on the theoretical sounding curve for the given distribution of resistivity with depth. These points are given to show how well the interpreted model fits the shifted-digitized curve.

4) A tabulation of the interpreted depths in meters and the interpreted resistivities in ohm-m.

Soundings $1,3,18$, and 19 had cusps that were obviously caused by cultural factors or current leakage. These cusps were manually smoothed before interpretation. Soundings 2, 4, 5, 12, 15, 16, 17, 20 and 21 had cusps and other features including rapid decreases of apparent resistivity from a maximum on the sounding curve. Such features were manually smoothed so that the inversion program could better fit the undistorted portions of the soundings. Smoothed soundings have "-S" designations after the title on the sounding interpretation plots in the appendix. Sounding 7 was not expanded to the originally planned $A B / 2$ spacing due to the effects of a buried telephone cable.

\section{GEOELECTRIC CROSS SECTION}

Figure 2 shows a geoelectric cross section constructed from the interpretation of the Schlumberger sounding data. The figure consists of two parts, a nonvertically exaggerated cross section, and the same cross section vertically exaggerated four times. The cross sections were generated in a manner similar to that described by Bisdorf (1982, pages 5 to 7 ). On the right hand side of the figure a scale is presented which relates interpreted resistivities with shades of gray. Darker shades indicate higher resistivities and lighter shades indicate lower resistivities. Triangles at the top of the cross sec- 


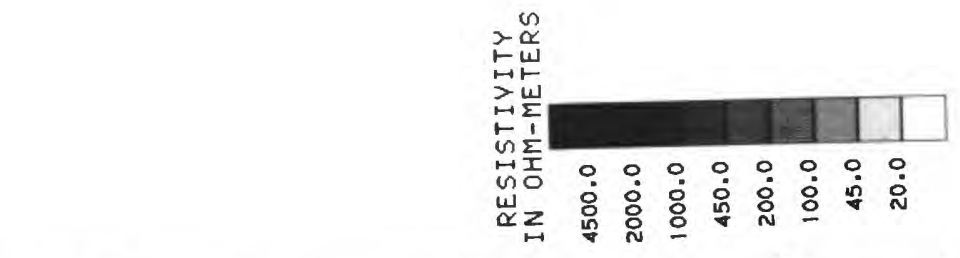

w
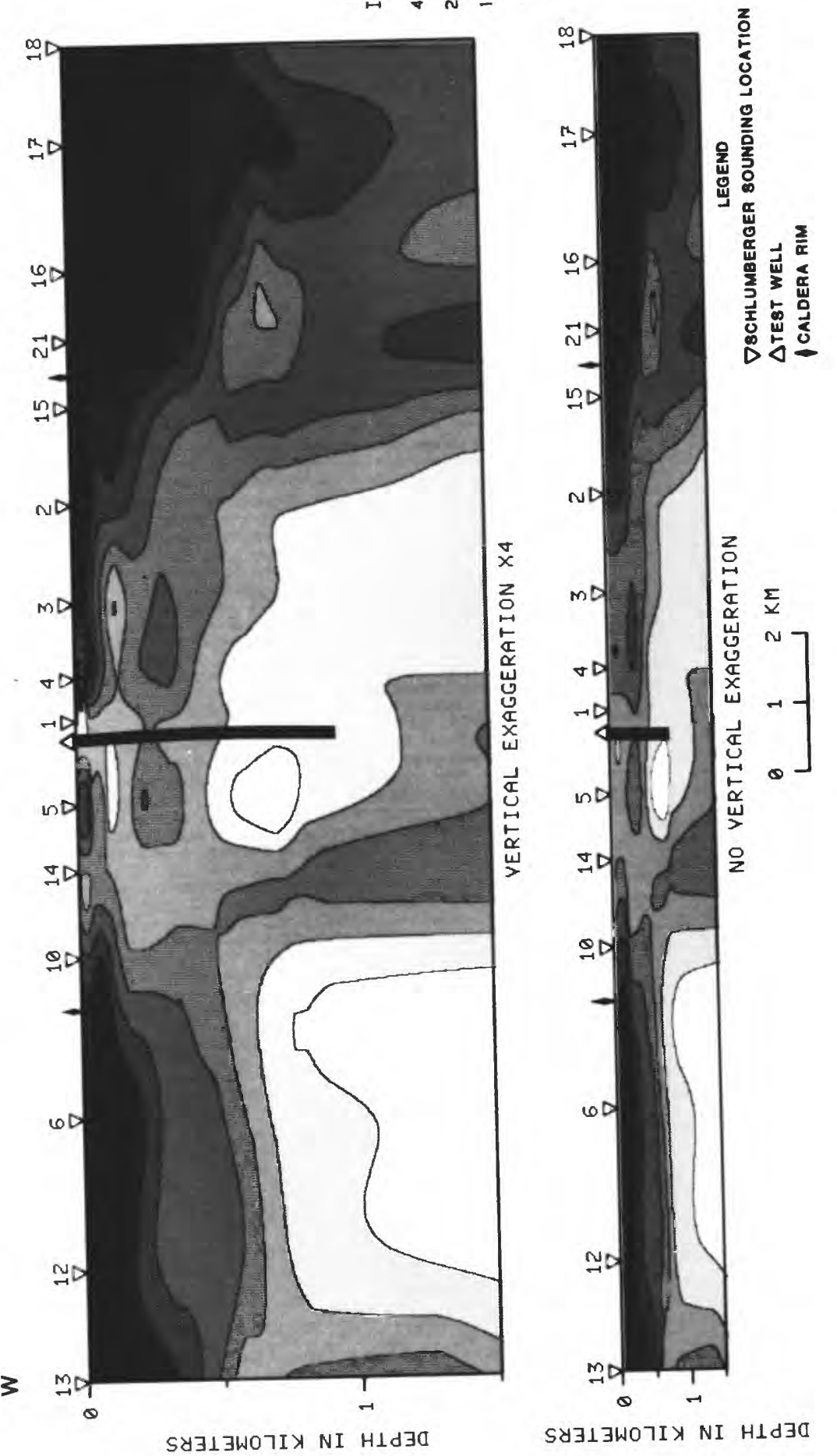

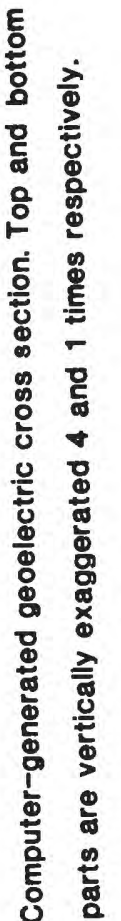

술 
tions indicate sounding locations and the numbers above the triangles are the sounding numbers.

Two factors need to be considered when interpreting the cross section. The first factor is that, due to the nature of the interpolation techniques used, anomalies not based on existing data may be generated between sounding locations. For example, between soundings 16 and 17 in the upper $100 \mathrm{~m}$ a lower resistivity area is shown. This area is very much lower than the resistivities under soundings 16 and 17 would dictate. The second factor is the limited resolution of the plotter which produced the original plots. Because of its fixed resolution the $\mathrm{xl}$ and $\mathrm{x}^{4}$ cross sections were generated with a different number of vertical points. Therefore the $\mathrm{xl}$ cross section is only presented to show the layered structure of the cross section and the $x^{4}$ cross section is presented to show the detail.

On the top of the cross section the approximate location of the caldera rim is shown. The location of the USGS test well described by Samme1 (1981) is shown where it projects onto the cross section. The well is located about $1 / 4$ mile southeast of this location (fig. 1).

The generalized lithologic $\log$ of the test well (Sammel, 1981) indicates that the intermediate resistivity, 45 to 1000 ohm-m, materials could correspond to tuffs, breccias, rhyolite flows, and lacustrine or fluviatile sediments. In the vicinity of sounding one, at a depth of about $600 \mathrm{~m}$, the main rock type changes to dacite and basalt flows. Generally these rock types exhibit higher resistivities than the 45 ohm-m (or lower) value indicated by the interpretation of soundings 4, 5, and 1 . The low resistivities could be indicative of a significant amount of alteration, large quantities of hot or saline water, or both. The temperature profile for the test well indicates an increase in temperature at about $680 \mathrm{~m}$, thus the low resistivity zone seems to 
correlate with the increasing temperature. A larger low resistivity zone at a depth of about $700 \mathrm{~m}$ is present under soundings 10, 6, and 12. The resistivity cross section does not indicate a hydrologic connection between the low resistivity areas in the upper $1.5 \mathrm{~km}$, but this zone probably contains hot water.

High resistivity material $(>1000 \mathrm{ohm}-\mathrm{m})$ is present in the upper $600 \mathrm{~m}$ along the flanks of the volcano, mostly outside the caldera. This material most likely consists of scoria and basalt or andesite flows. A thin $(<100 \mathrm{~m})$ layer of this material extends into the caldera from the east.

\section{SUMMARY}

The USGS test well has shown the presence of hot water in the Newberry Caldera. Schlumberger sounding interpretations correlate well with the drill hole indications of elevated borehole temperatures. Low resistivity zones can be assumed to be related to the presence of hot water or alteration zones. Another low resistivity zone not necessarily hydrologically related to the zone near the test well is present to the west and could contain significant amounts of hot water.

High resistivity material up to $600 \mathrm{~m}$ thick was detected on the flanks of the volcano. High-resistivity materials do not occur in such large thicknesses inside the caldera. The high-resistivity unit either does not occur inside the caldera, or it has been altered.

\section{ACKNOWLEDGMENT}

This work was funded in part by the Department of Energy under the Interagency Agreement DE-AI01-79RA50294. Permission to conduct the field work was granted by the Dept. of Agriculture, Forest Service, Deschutes National Forest, Bend, Oregon. The field work was accomplished with the help of Dean Schoenthaler, R. Grette, D. Piper, M. Seals and D. Churchill. 
REFERENCES

Bisdorf, R. J., 1982, Schlumberger sounding investigations in the Date Creek Basin, Arizona: U.S. Geological Survey Open-File Report 82-953, 54 p. Bisdorf, R. J., and Zohdy, A. A. R., 1979, Geoelectric investigations with Schlumberger soundings near Brunswick, Georgia: U.S. Geological Survey Open-File Report 79-1551, 125 p.

Ghosh, D. P., 1971, Inverse filter coefficients for the computation of apparent resistivity standard curves over horizontally stratified earth: Geophysical Prospecting [Netherlands], v. 19, no. 4, p. 769-775.

McLeod, N. S., Sherrod, D. R., Chitwood, L. A., and McKee, E. H., 1981, Newberry Volcano, Oregon: Guide to some Volcanic Terranes in Washington, Idaho, Oregon and Northern California (D. A. Johnston and J. DonellyNolan, eds), U.S. Geological Survey Circular 838, p. 85-103.

0'Neill, D. J., 1975, Improved linear filter coefficients for application in apparent resistivity computations: Bulletin Australian Society Exploration Geophysics, v. 6, no. 4, p. 104-109.

Samme1, E. A., 1981, Results of test drilling at Newberry Volcano, Oregon: Geothermal Resources Council Bull., Dec. 1981.

Zohdy, A. A. R., 1973, A computer program for the automatic interpretation of Schlumberger sounding curves over horizontally stratified media: available only from U.S. Dept. of Commerce, Nat'1. Tech. Inf. Service, Springfield, Va. 22161, as U.S. Geological Survey Report USGS-GD-74-017, PB232 703.

, 1975, Automatic interpretation of Schlumberger sounding curves using modified Dar Zarrouk functions: U.S. Geological Survey Bul1. 1313-E, 39p. Zohdy, A. A. R., and Bisdorf, R. J., 1982, Schlumberger soundings in the Medicine Lake Area, California, U.S. Geological Survey Open-File Report $82-887,162$ p. 
APPENDIX 


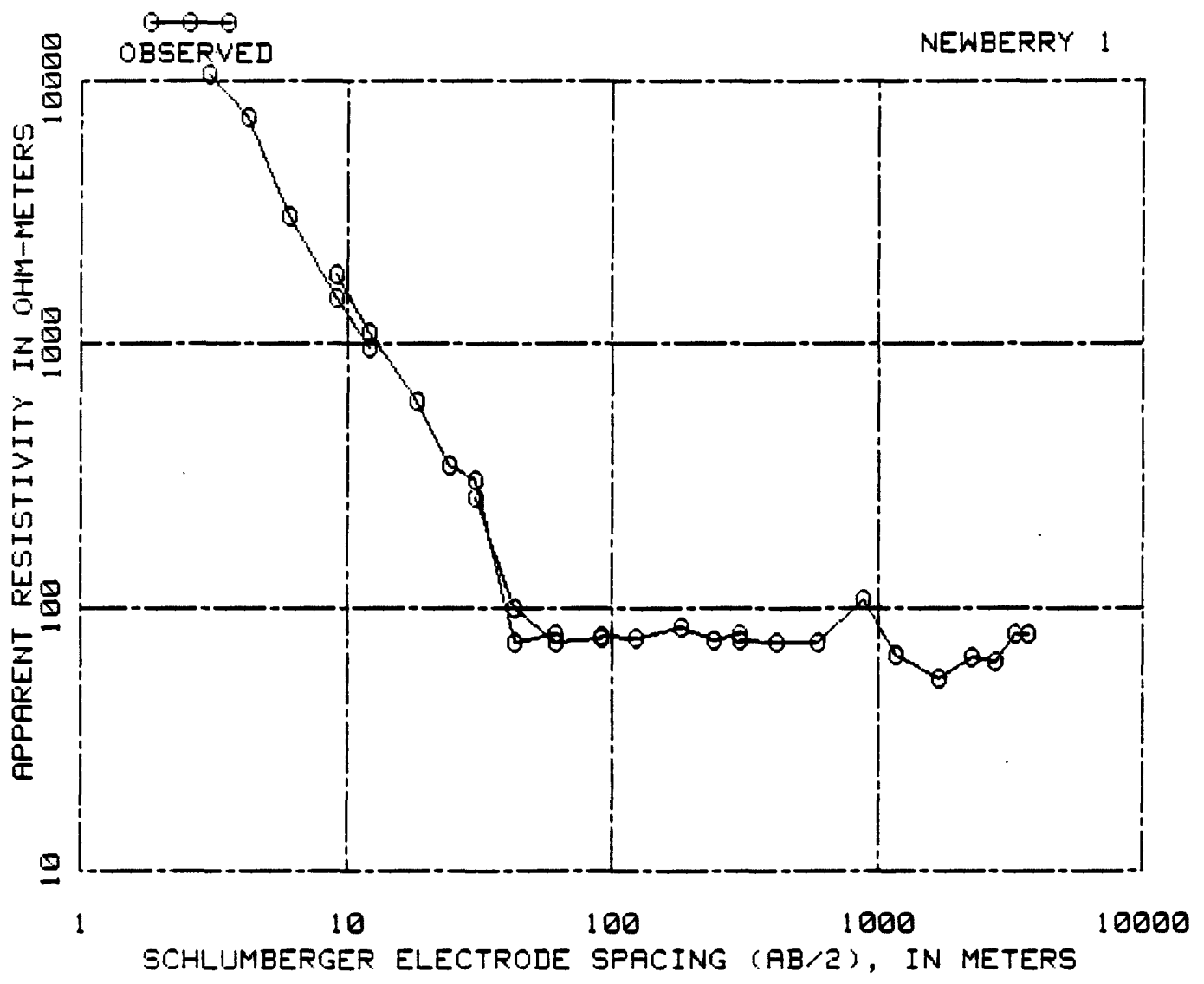

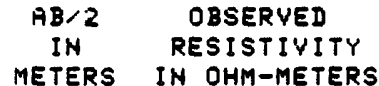

91.44

121.92

182.88

243.84

304.80

304.80

419.71

593.45

889.41

889.41

1181.71

1692.86

2267.71

2769.11

3297.63

3713.68
78.00

76.00

84.00

75.00

79.00

75.00

73.00

73.00

108.00

108.00

66.00

54:00

65.00

62.00

79.00

79.00 


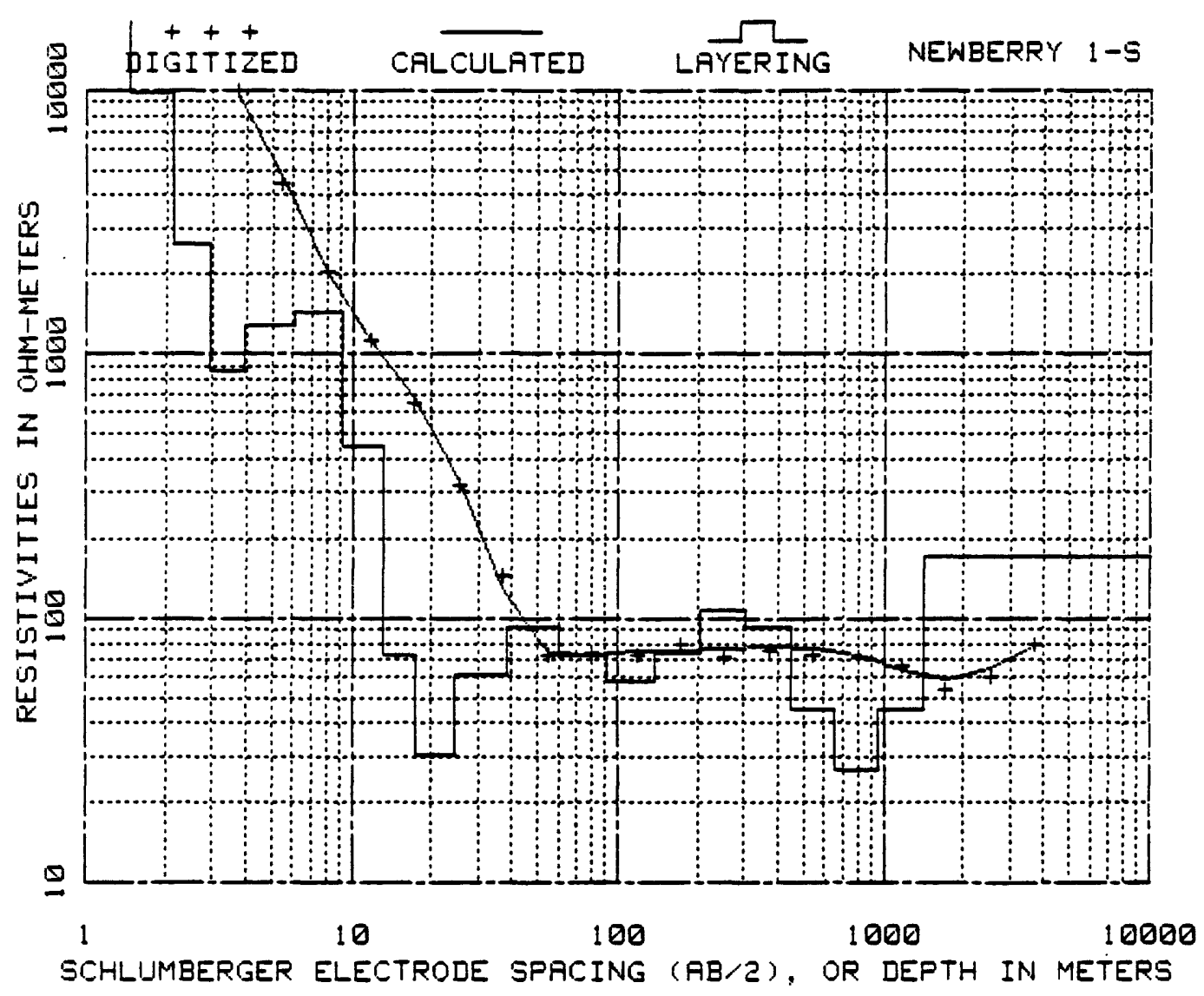

$\begin{array}{cr}\text { INTERPRETED } & \text { INTERPRETED } \\ \text { DEPTH } & \text { RESISTIVI } \\ \text { IN METERS } & \text { IN OHM-METER } \\ & \\ .21 & 20345.26 \\ .31 & 19984.27 \\ .46 & 19548.74 \\ .68 & 29255.62 \\ .99 & 22520.22 \\ 1.46 & 20299.58 \\ 2.11 & 9751.39 \\ 2.90 & 2634.53 \\ 3.97 & 858.50 \\ 6.03 & 1288.29 \\ 3.16 & 1422.66 \\ 13.18 & 448.61\end{array}$

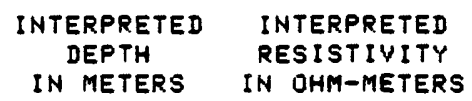

17.37

24.31

38.67

60.23

91.80

137.85

206.13

301.62

447.97

657.04

953.39

1418.54

1001417.54
72.29

30.66

60.58

91.58

72.48

57.61

73.00

106.31

92.63

45.12

26.55

45.30

171.69 


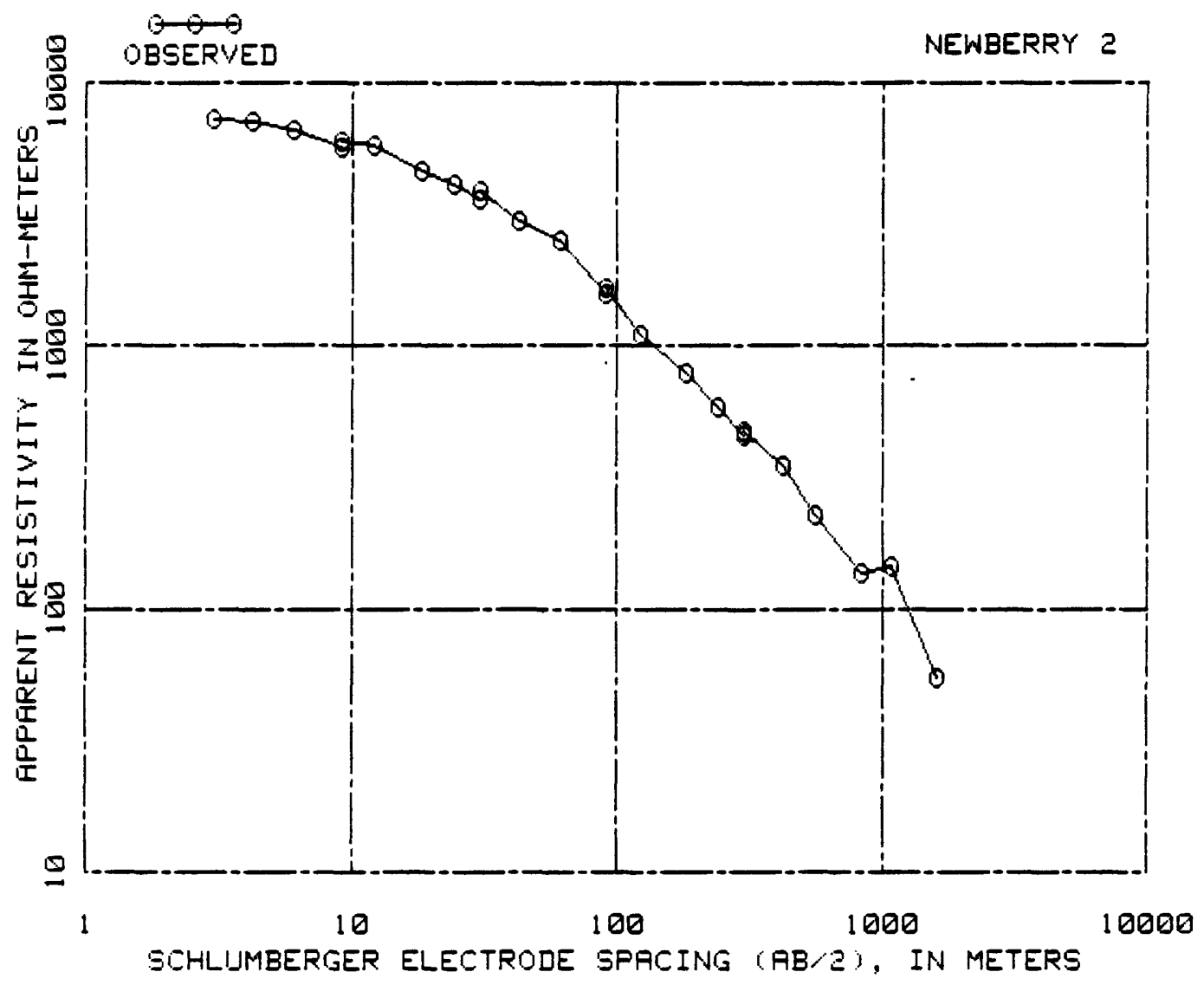

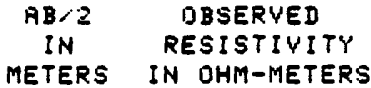

$91.44 \quad 1580.00$

$91.44 \quad 1660.00$

$121.92 \quad 1100.00$

$182.88 \quad 790.00$

$243.84 \quad 580.08$

$304.80 \quad 460.00$

$304.30 \quad 473.00$

$426.72 \quad 350.00$

$565.48 \quad 228.00$

$834.85 \quad 136.00$

$834.35 \quad 133.00$

$1087.53 \quad 146.00$

$1600.50 \quad 55.00$ 


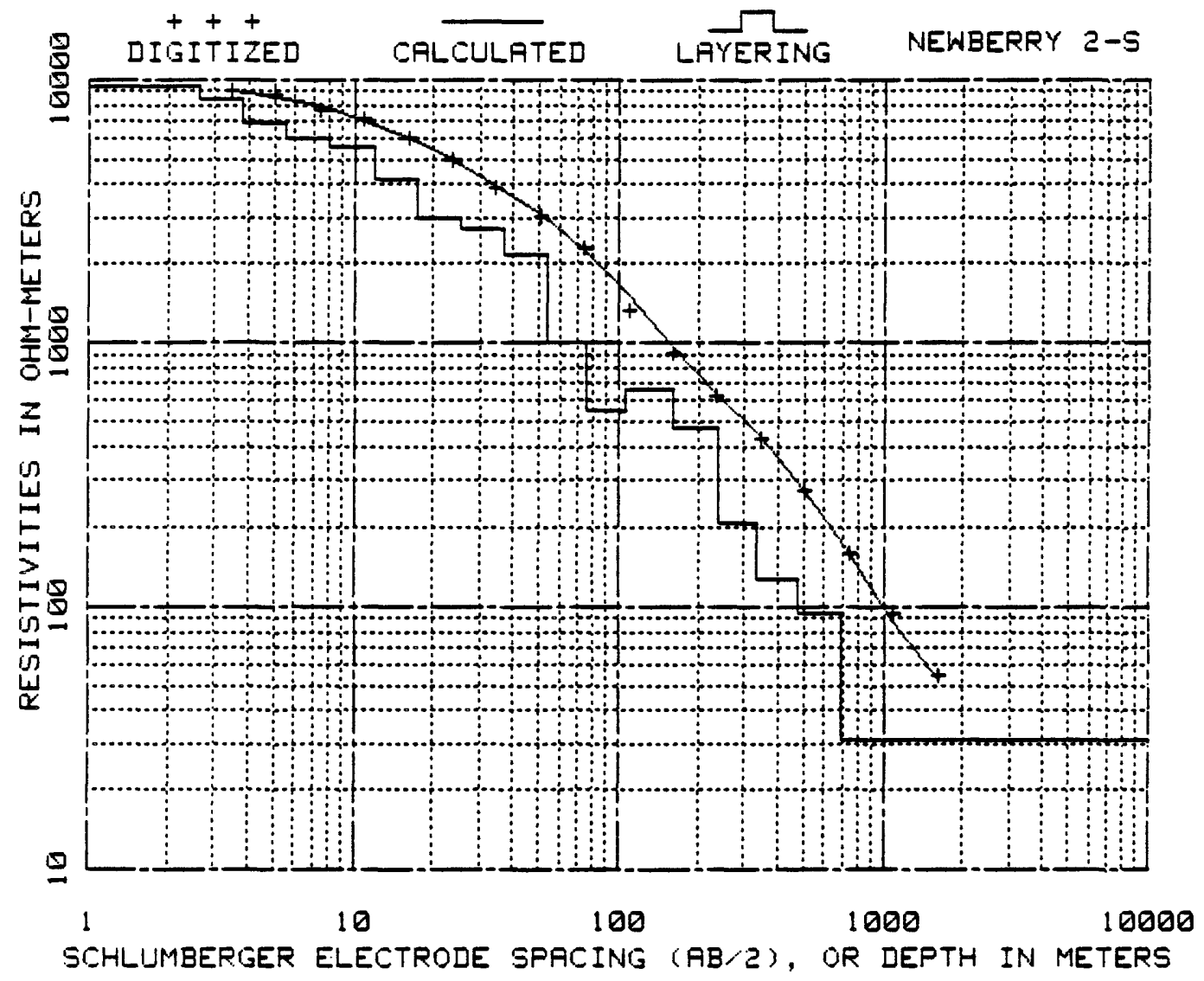

$\begin{array}{cc}\text { INTERPRETED } & \text { INTERPRETED } \\ \text { DEPTH } & \text { RESISTIVITY } \\ \text { IN METERS } & \text { IN OHM-METERS }\end{array}$

17.34
25.13
36.79
53.80
75.76
106.16
159.18
235.46
331.88
473.57
693.68
692.68

17.34

9357.84

9355.97

9346.42

9339.19

9379.98

9493.39

9423.10

8433.89

6787.45

5974.26

5590.59

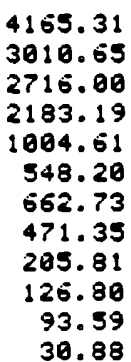




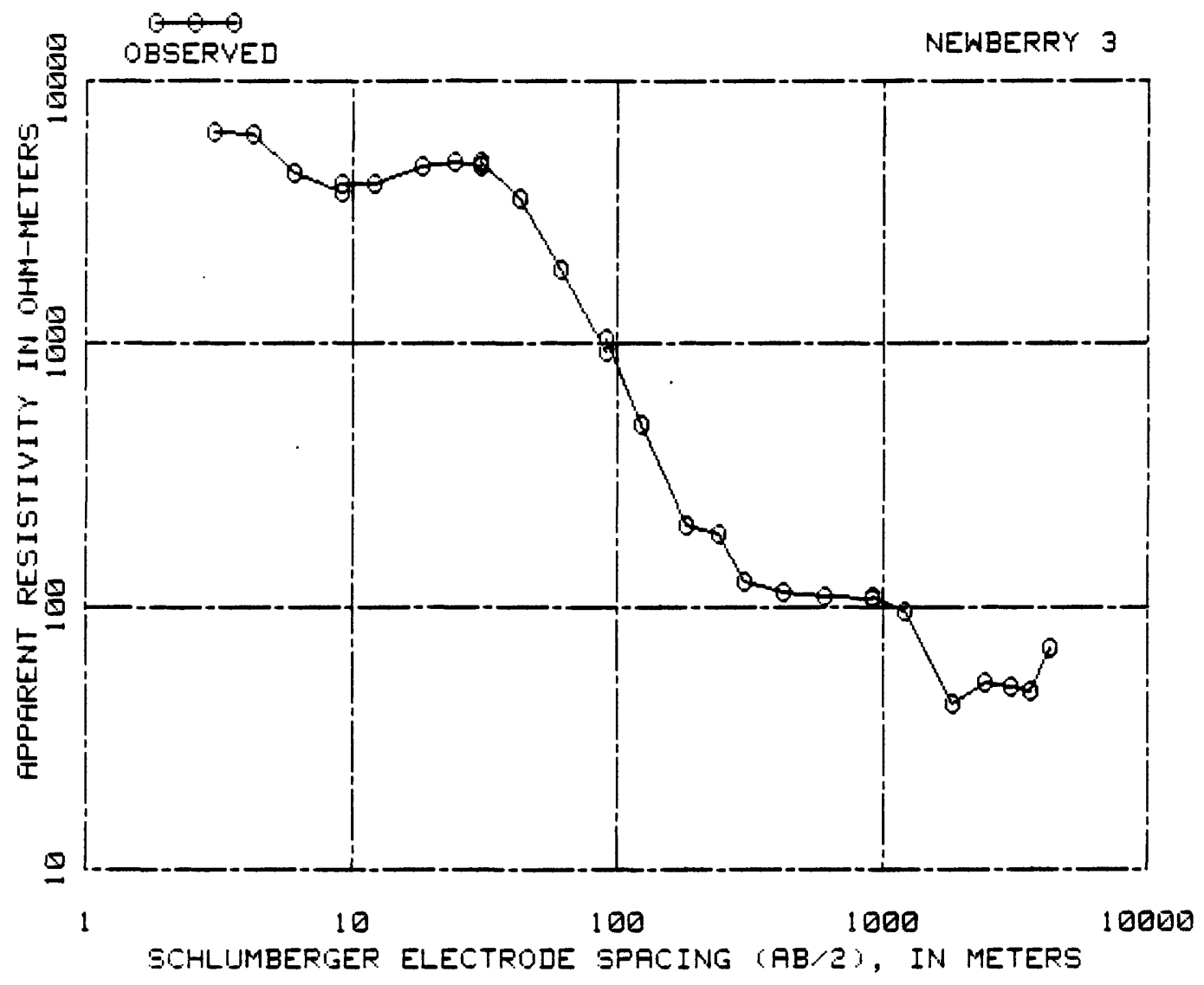

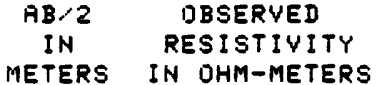

$\begin{array}{rr}3.05 & 6350.00 \\ 4.27 & 6210.00 \\ 5.10 & 4400.00 \\ 7.14 & 3760.00 \\ 9.14 & 4050.00 \\ 12.19 & 4050.00 \\ 18.29 & 4650.00 \\ 24.38 & 4900.00 \\ 30.48 & 4700.00 \\ 30.43 & +900.00 \\ 42.67 & 3550.00 \\ 60.96 & 1900.00 \\ 91.44 & 940.00 \\ 71.44 & 1050.00\end{array}$

$\begin{array}{cc}\text { AB } / 2 & \text { OBSERVED } \\ \text { IN } & \text { RESISTIVITY } \\ \text { METERS } & \text { IN OHM-METERS }\end{array}$

121.92

182.38

243.34

304.80

304.30

426.72

609.60

914.40

714.40

1217.20

1828.80

2438.40

3048.00

3657.60

4267.20
492.00

203.00

187.00

124.00

125.00

114.00

110.00

107.00

110.00

95.00

$+3.00$

52.00

50.00

48.00

69.00 


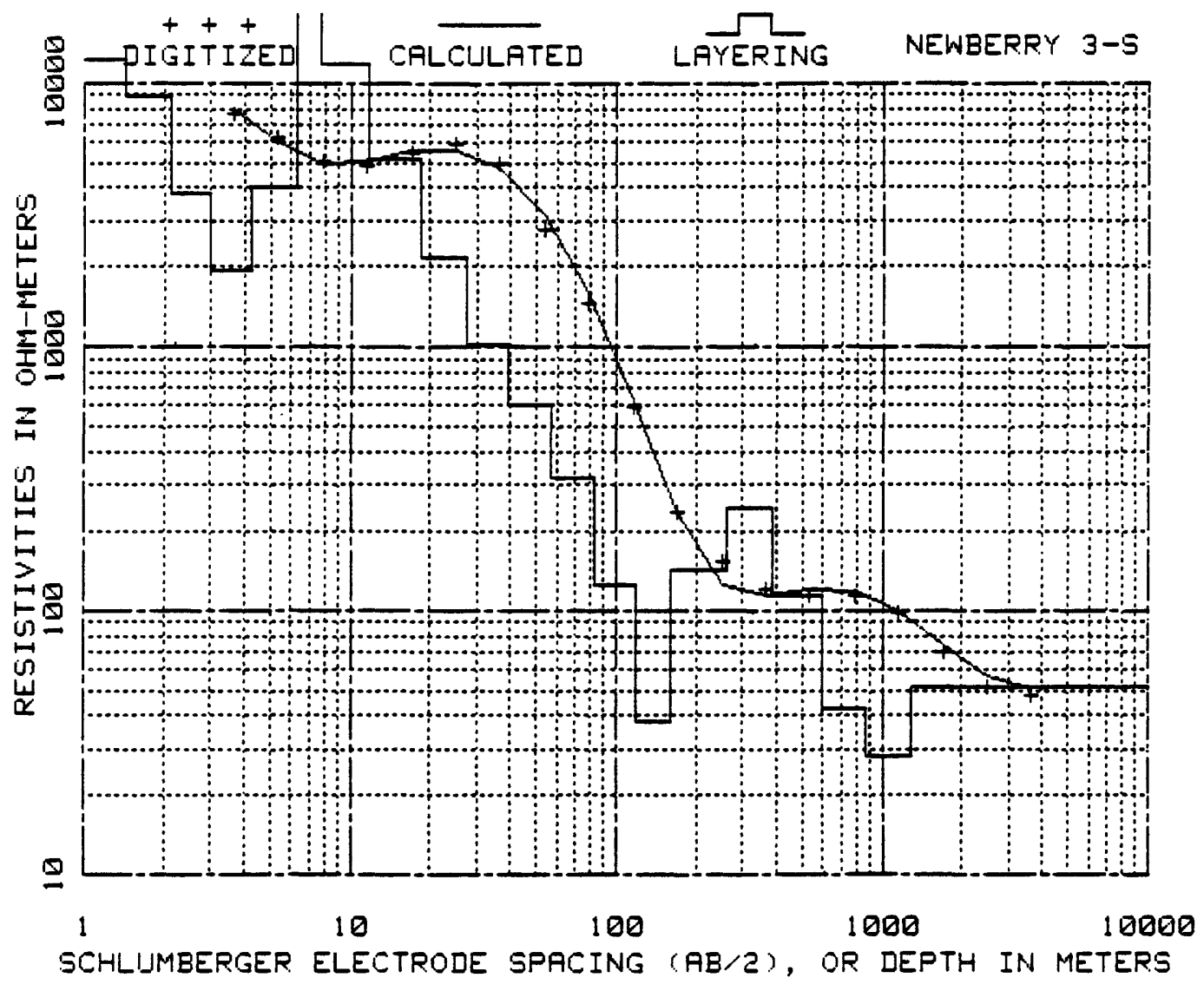

$\begin{array}{cc}\text { INTERPRETED } & \text { INTERPRETED } \\ \text { DEPTH } & \text { RESISTIVITY } \\ \text { IN METERS } & \text { IN OHM-METERS }\end{array}$

$\begin{array}{rr}18.35 & 5174.14 \\ 27.20 & 2158.36 \\ 39.31 & 1026.42 \\ 57.26 & 604.16 \\ 83.07 & 319.99 \\ 117.42 & 124.37 \\ 161.40 & 38.46 \\ 260.04 & 142.59 \\ 389.28 & 247.52 \\ 599.26 & 114.32 \\ 871.61 & 42.81 \\ 1277.30 & 28.27 \\ 1001276.30 & 51.64\end{array}$




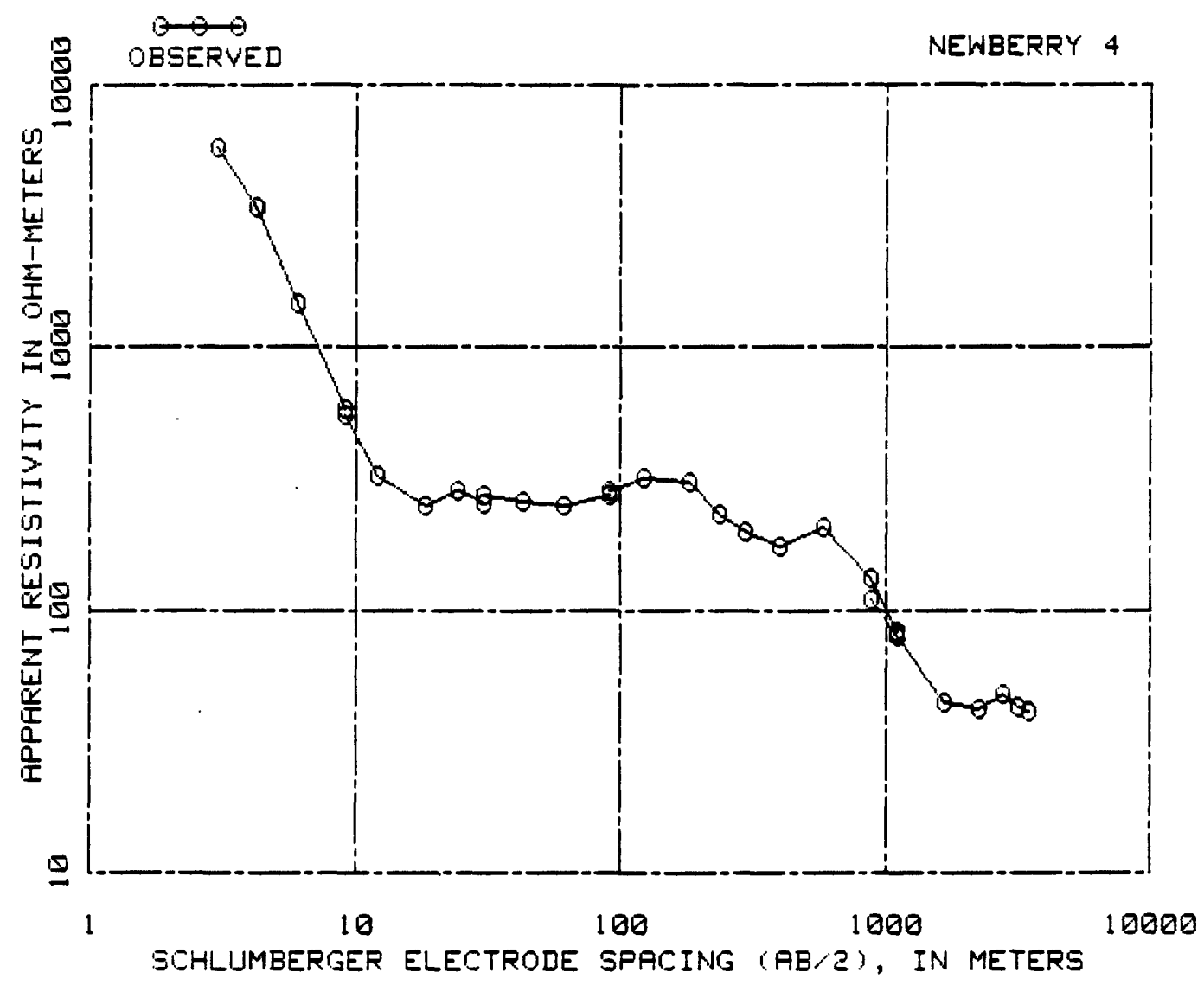

$\begin{array}{cr}\text { AB/2 } & \text { DBSERVED } \\ \text { IN } & \text { RESISTIVIT } \\ \text { METERS } & \text { IN OHM-METE } \\ & \\ 3.05 & 5790.00 \\ 4.27 & 3400.00 \\ 5.10 & 1475.00 \\ 9.14 & 580.00 \\ 9.14 & 555.00 \\ 12.19 & 326.00 \\ 18.29 & 252.00 \\ 24.38 & 285.00 \\ 30.48 & 254.00 \\ 30.48 & 277.00 \\ 42.67 & 260.00 \\ 50.96 & 250.00 \\ 91.44 & 275.00 \\ 91.44 & 285.00 \\ 121.92 & 320.00\end{array}$

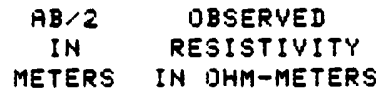

182.88

237.74

295.05

295.05

404.16

589.48

881.79

1107.34

881.79

1107.34

1689.51

2246.99

2804.16

3197.05

3514.34
310.00

231.00

198.00

199.00

174.00

208.00

133.00

83.00

110.00

79.00

$+4.00$

42.00

48.00

43.00

41.00 


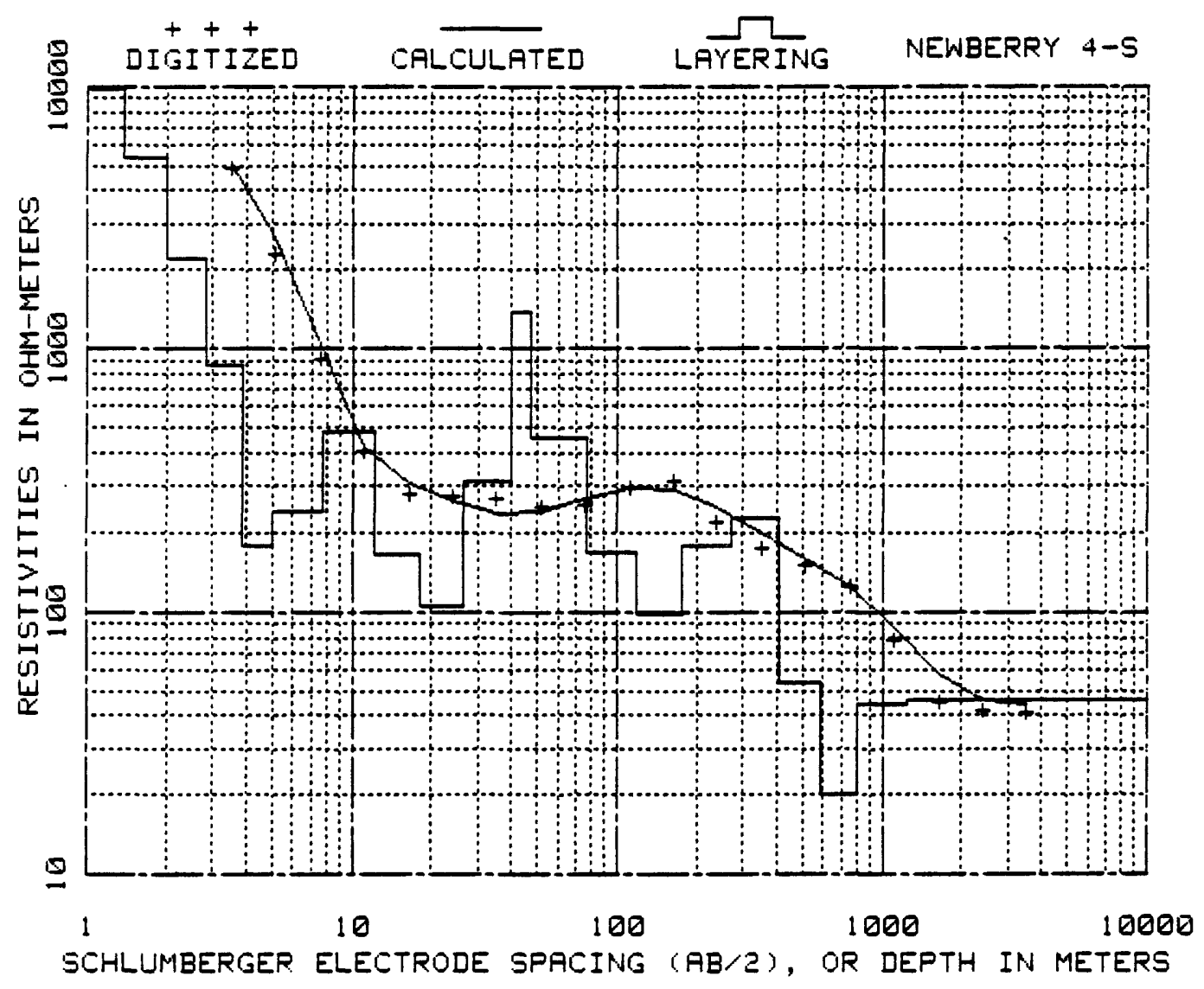

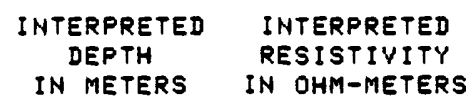

17.93
26.40
39.53
47.57
76.60
118.11
176.65
271.49
408.38
583.56
812.17
1250.22
1249.22
165.38

104.69

311.18

1384.02

453.74

167.43

97.44

177.19

226.68

53.08

20.11

44.30

45.64 


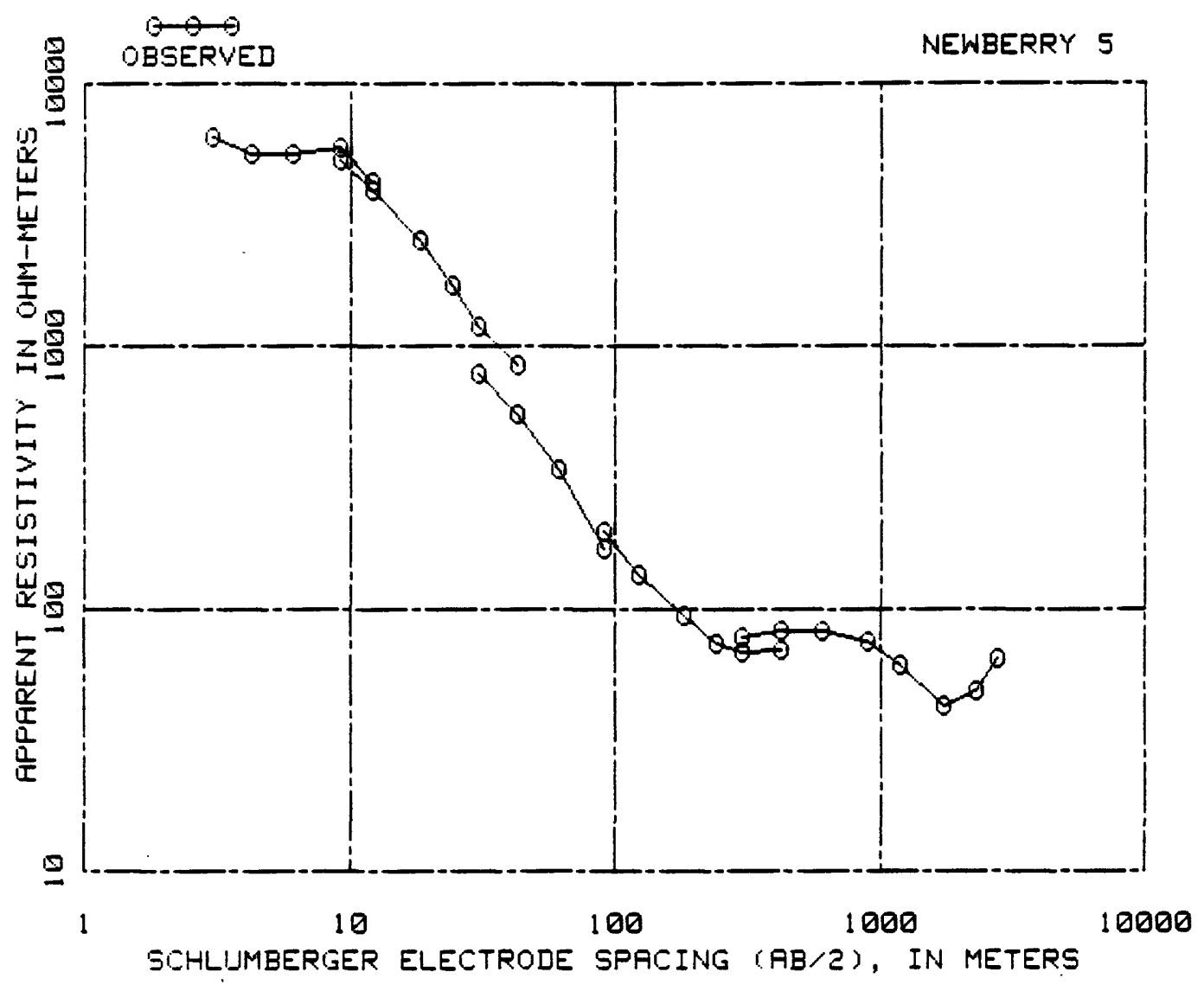

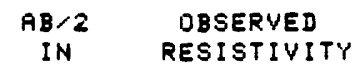

METERS IN DHM-METERS

$\begin{array}{rr}3.05 & 6180.00 \\ 4.27 & 5400.00 \\ 5.10 & 5400.00 \\ 7.14 & 5700.00 \\ 12.19 & 4180.00 \\ 9.14 & 5100.00 \\ 12.19 & 3920.00 \\ 18.29 & 2510.00 \\ 24.38 & 1700.00 \\ 30.48 & 1200.00 \\ 42.67 & 950.00 \\ 30.48 & 780.00 \\ 42.67 & 550.00 \\ 50.96 & 340.00\end{array}$

AB/2 DBSERYED

IN RESISTIVITY

METERS IN DHM-METERS

$\begin{array}{rr}91.44 & 170.00 \\ 91.44 & 194.00 \\ 121.92 & 135.00 \\ 182.88 & 94.00 \\ 243.84 & 74.00 \\ 304.80 & 68.00 \\ 426.72 & 70.00 \\ 304.80 & 78.00 \\ 426.72 & 82.00 \\ 609.60 & 82.00 \\ 905.56 & 75.00 \\ 1196.95 & 61.00 \\ 1748.33 & 43.00 \\ 2305.20 & 49.00 \\ 2803.86 & 65.00\end{array}$




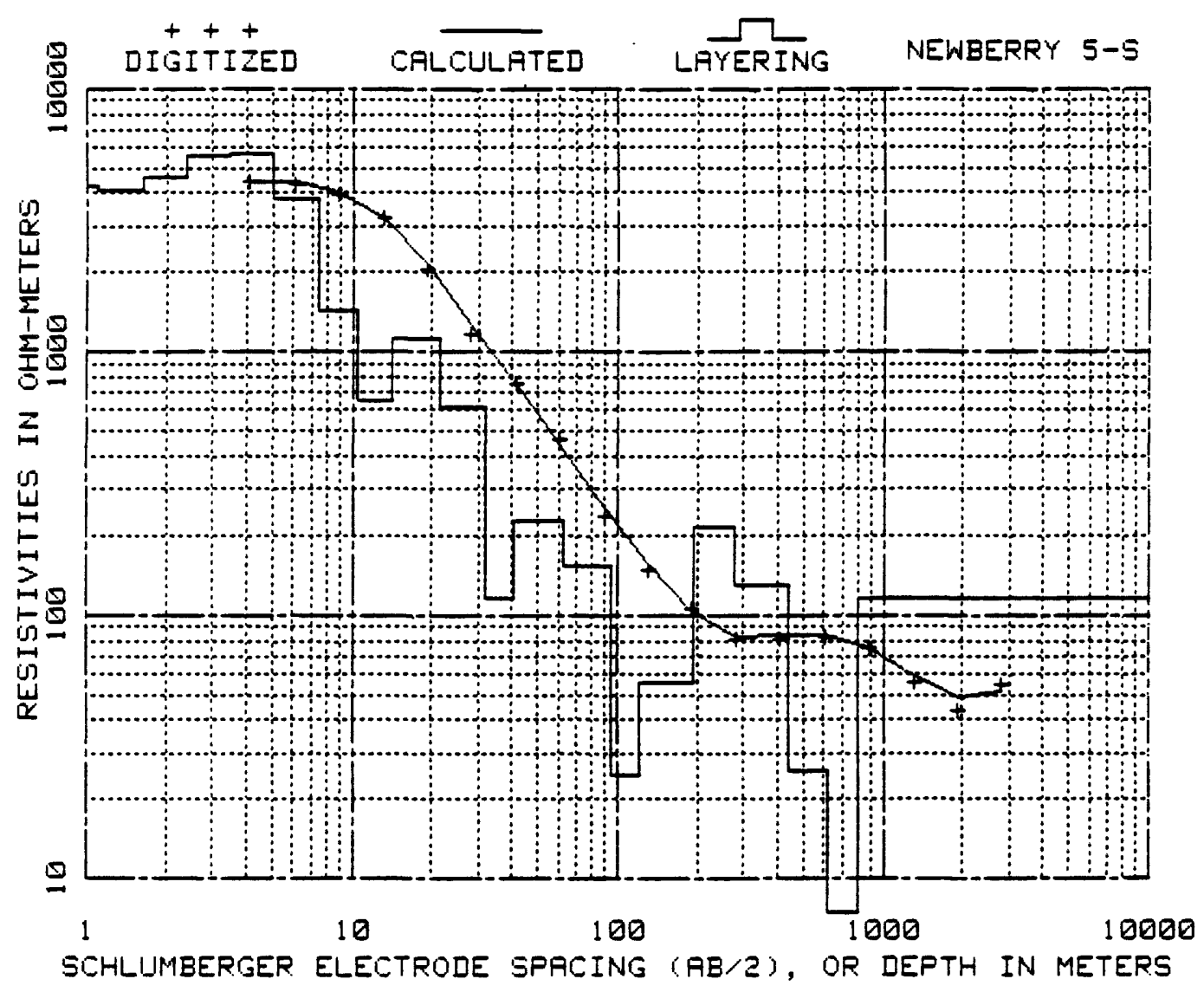

$\begin{array}{cr}\begin{array}{c}\text { INTERPRETED } \\ \text { DEPTH }\end{array} & \begin{array}{r}\text { INTERPRET } \\ \text { RESISTIVI } \\ \text { IN METERS }\end{array} \\ & \\ & \\ .24 \text { OHM-MET } & \\ .35 & 4266.42 \\ .51 & 4235.73 \\ .75 & 4271.50 \\ 1.10 & 4323.23 \\ 1.62 & 4234.87 \\ 2.37 & 4138.82 \\ 3.46 & 4598.42 \\ 5.87 & 5514.84 \\ 7.44 & 5648.47 \\ 10.39 & 3898.38 \\ 14.22 & 1433.63 \\ & 552.72\end{array}$

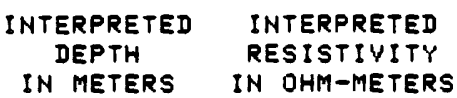

21.61
31.67
40.48
62.78
94.63
120.56
191.50
276.43
433.09
616.36
809.60
308.60

1120.55

621.38

115.07

227.77

154.54

24.80

55.54

213.86

130.22

25.37

7.37

115.71 


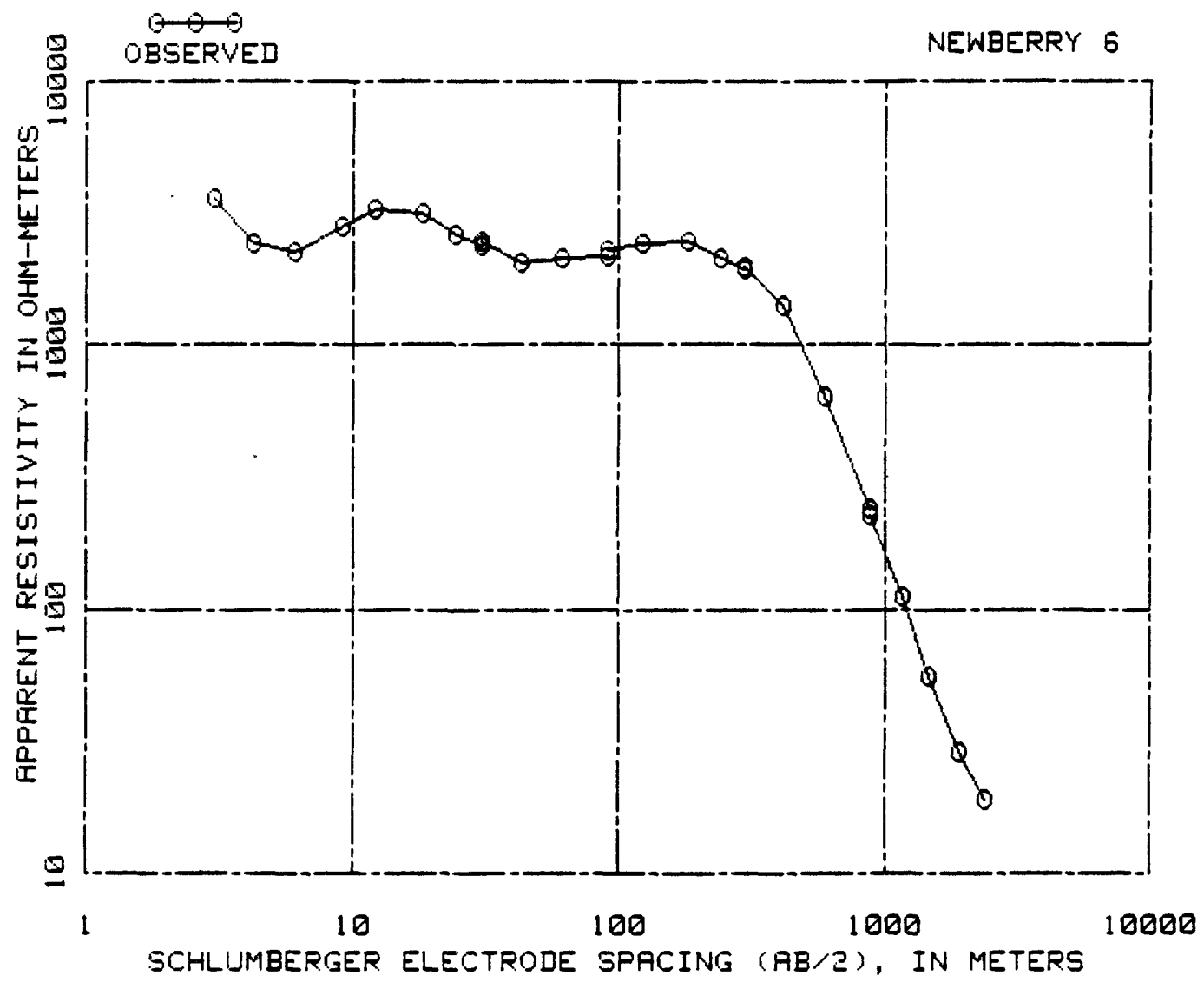

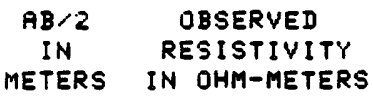

91.44

121.92

182.88

239.88

300.23

300.23

418.80

599.54

891.54

891.54

1177.75

1473.71

1908.96

2383.54
2280.00

2450.00

2500.00

2133.00

1950.08

1969.00

1408.00

638.00

239.00

229.00

112.00

56.00

29.00

19.00 


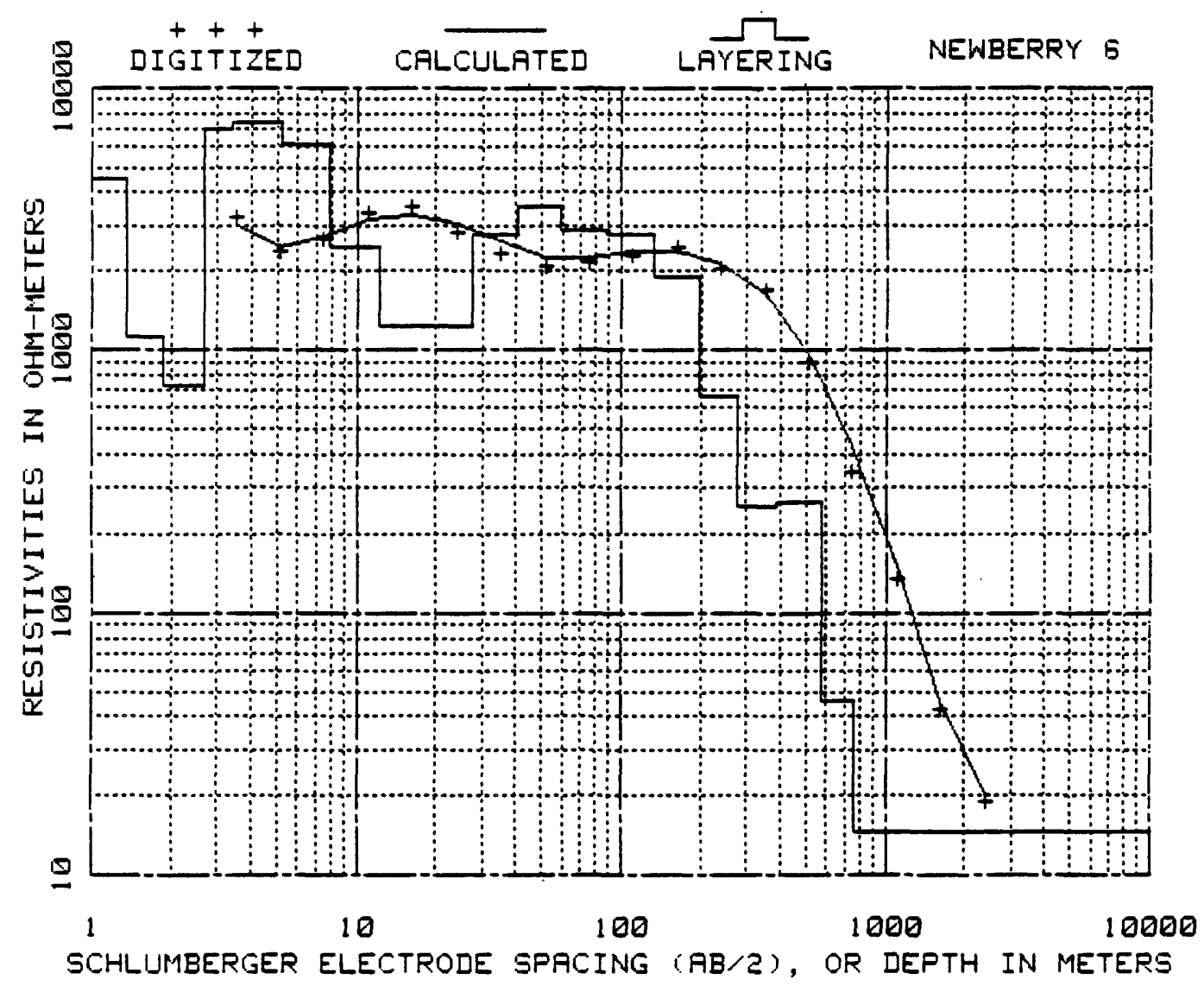

$\begin{array}{cc}\begin{array}{c}\text { INTERPRETED } \\ \text { DEPTH }\end{array} & \begin{array}{r}\text { INTERPRET } \\ \text { RESISTIVI } \\ \text { IN METERS } \\ \text { IN OHM-MET }\end{array} \\ & \\ .20 & 6896.26 \\ .30 & 6822.36 \\ .43 & 6518.11 \\ .64 & 7112.80 \\ .93 & 8999.57 \\ 1.36 & 4528.78 \\ 1.86 & 1134.97 \\ 2.65 & 727.81 \\ 3.39 & 6938.21 \\ 5.22 & 7391.98 \\ 7.97 & 6117.01 \\ 12.23 & 2487.04\end{array}$

$\begin{array}{cc}\text { INTERPRETED } & \text { INTERPRETED } \\ \text { DEPTH } & \text { RESISTIVITY } \\ \text { IN METERS } & \text { IN OHM-METERS }\end{array}$

$\begin{array}{rr}18.02 & 1228.46 \\ 27.10 & 1233.07 \\ 40.25 & 2753.92 \\ 58.76 & 3561.65 \\ 88.38 & 2863.13 \\ 132.12 & 2790.28 \\ 195.81 & 1916.86 \\ 277.14 & 559.77 \\ 383.60 & 253.99 \\ 570.56 & 265.99 \\ 757.68 & 46.32 \\ 1000756.60 & 14.61\end{array}$



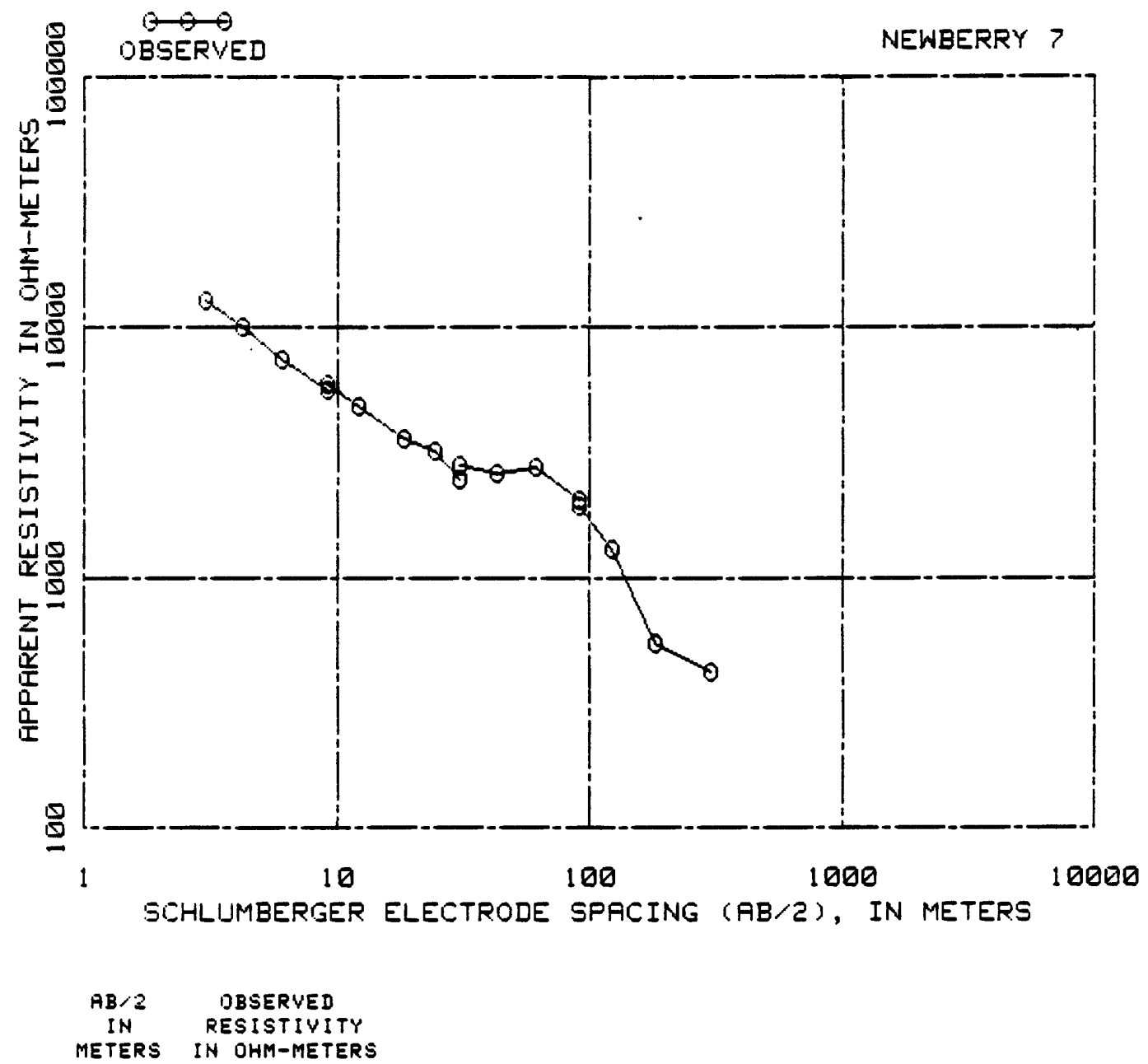

$\begin{array}{rr}3.05 & 12800.00 \\ 4.27 & 10000.00 \\ 6.10 & 7450.00 \\ 7.14 & 5650.00 \\ 9.14 & 5900.00 \\ 12.19 & 4800.00 \\ 18.29 & 3600.00 \\ 24.38 & 3200.00 \\ 30.48 & 2450.00 \\ 30.48 & 2800.00 \\ 42.67 & 2600.00 \\ 60.96 & 2760.00 \\ 91.44 & 2020.00 \\ 91.44 & 1910.00 \\ 121.92 & 1300.00 \\ 182.83 & 550.00 \\ 304.30 & 410.00\end{array}$




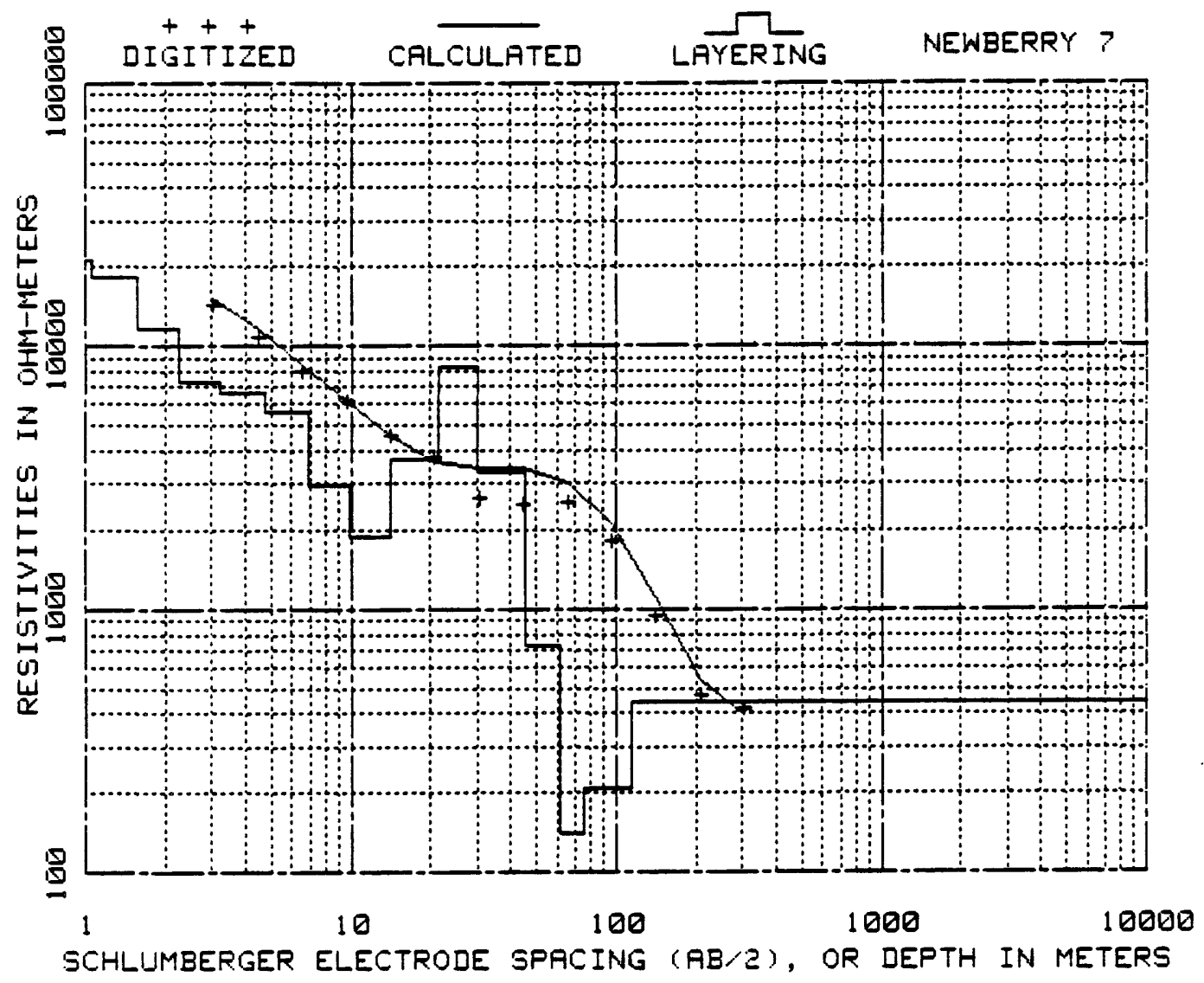

$\begin{array}{cr}\begin{array}{c}\text { INTERPRETED } \\ \text { DEPTH }\end{array} & \begin{array}{r}\text { INTERPRET } \\ \text { RESISTIVI } \\ \text { IN METERS } \\ \text { IN OHM-MET }\end{array} \\ & \\ .23 & 20704.94 \\ .34 & 20646.29 \\ .49 & 20623.52 \\ .72 & 20943.78 \\ 1.06 & 21191.84 \\ 1.56 & 18199.41 \\ 2.25 & 11577.78 \\ 3.23 & 7260.01 \\ 4.72 & 6609.55\end{array}$

$\begin{array}{cc}\text { INTERPRETED } & \text { INTERPRETED } \\ \text { DEPTH } & \text { RESISTIVITY } \\ \text { IN METERS } & \text { IN OHM-METERS }\end{array}$

$\begin{array}{rr}6.92 & 5632.57 \\ 9.88 & 2967.99 \\ 14.12 & 1898.79 \\ 21.41 & 3716.99 \\ 30.04 & 8383.42 \\ 45.47 & 3302.72 \\ 61.02 & 721.28 \\ 74.86 & 139.66 \\ 114.07 & 209.08 \\ 1000113.07 & 443.96\end{array}$




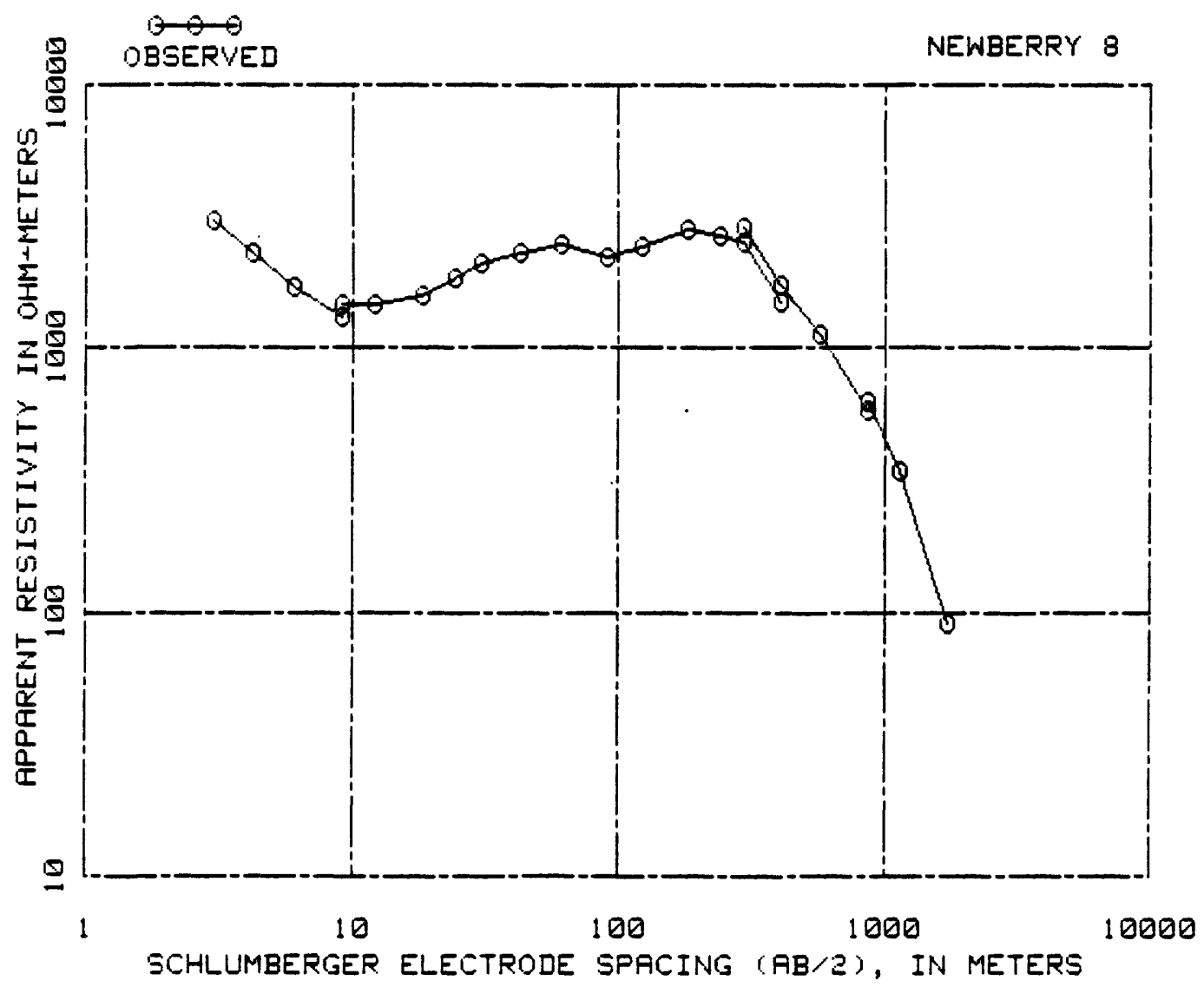

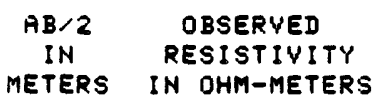

3.05

4.27

6. 10

9. 14

3.14

12. 19

18.29

24.38

30.48

30.48

42.67

60.96

91.44
3050.00

2280.00

1710.00

1300.08

1460.00

1450.00

1580.00

1820.00

2100.00

2110.00

2300.00

2500.00

2200.00

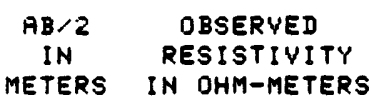

91.44

121.92

182.88

241.71

29.5 .66

406.91

295.66

406.91

579.42

865.02

865.02

1159.15

1753.51
2200.00

2420.00

2800.00

2665.08

2503.00

1502.00

2905.00

1739.00

1128.00

587.00

631.00

342.00

90.00 


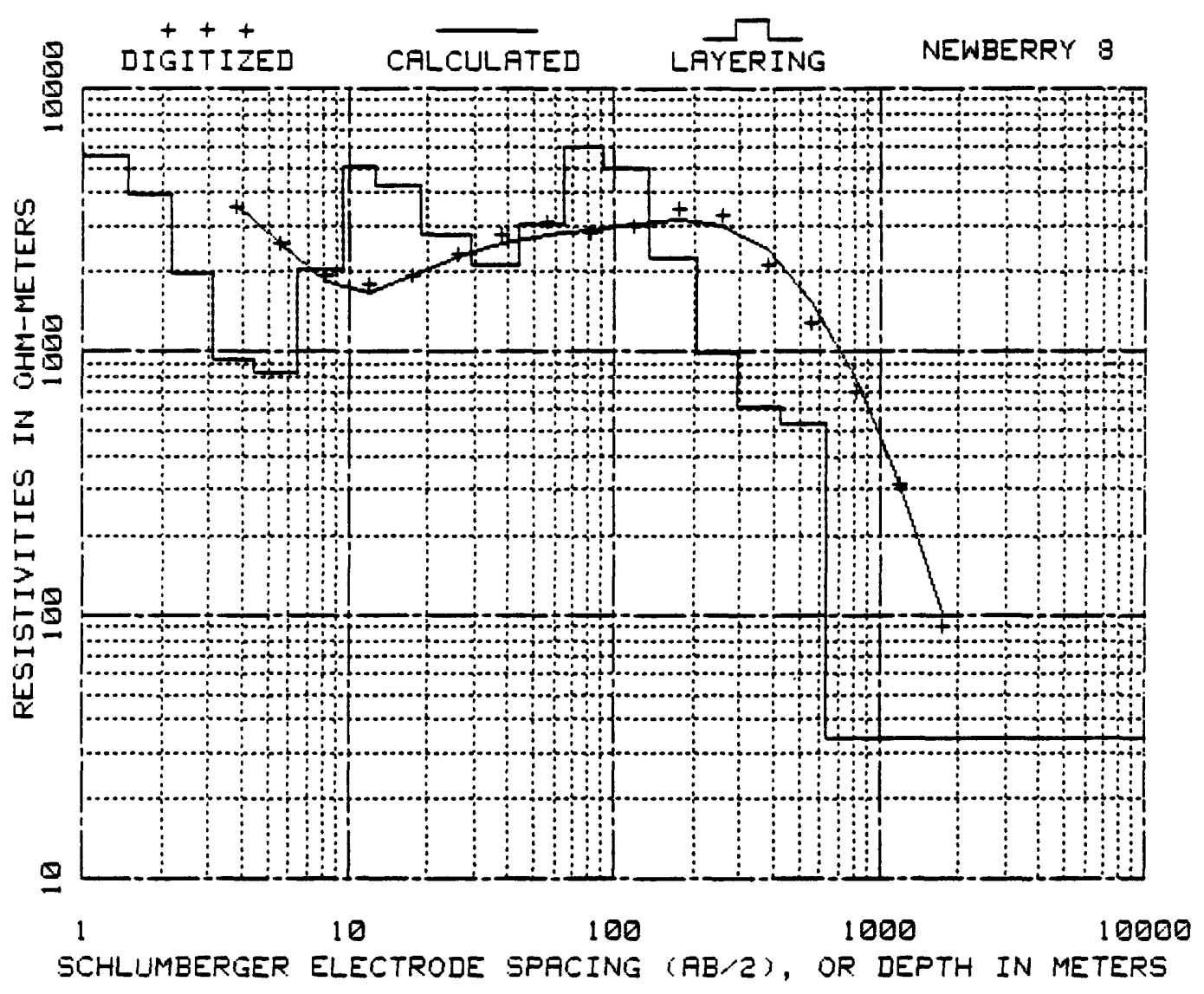

$\begin{array}{cc}\text { INTERPRETED } & \text { INTERPRETED } \\ \text { DEPTH } & \text { RESISTIVITY } \\ \text { IN METERS } & \text { IN OHM-METERS }\end{array}$

12.65

18.61

28.80

43.69

65.31

91.12

136.03

203.78

293.84

425.96

631.10

1000630.10
5047.45

4252.80

2750.16

2126.88

3070.76

6010.50

4947.36

2266.35

984.41

620.75

533.60

34.24 


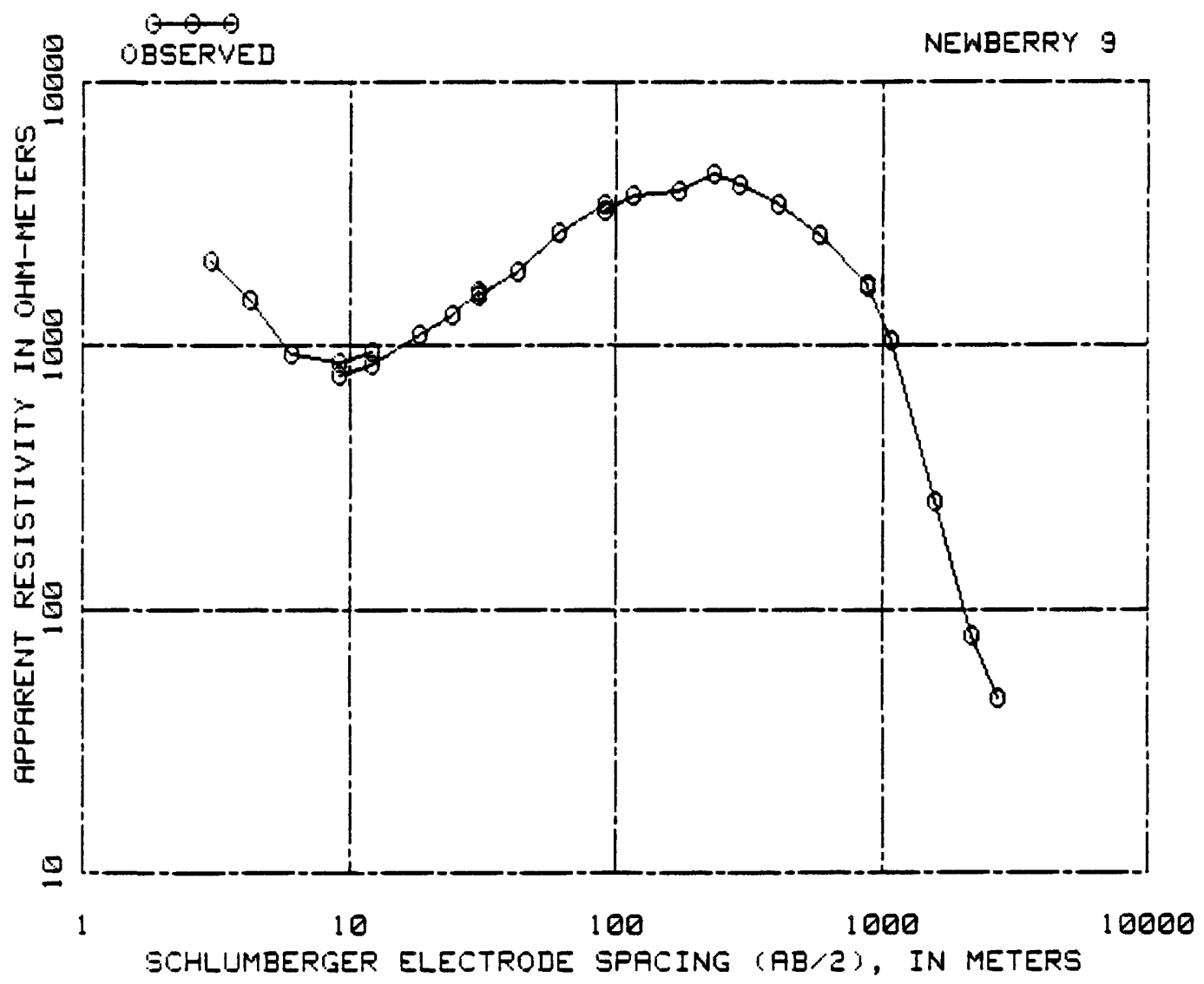

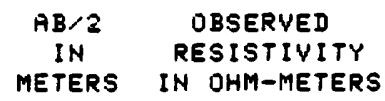

91.44

115.21

171.91

233.78

290.78

290.78

409.35

590.09

882.70

882.70

1088.14

1586.48

2174.14

2753.26
3200.00

3698.00

3847.08

4409.00

4059.00

4072.00

3415.00

2645.00

1692.00

1682.00

1034.00

257.00

79.00

46.00 


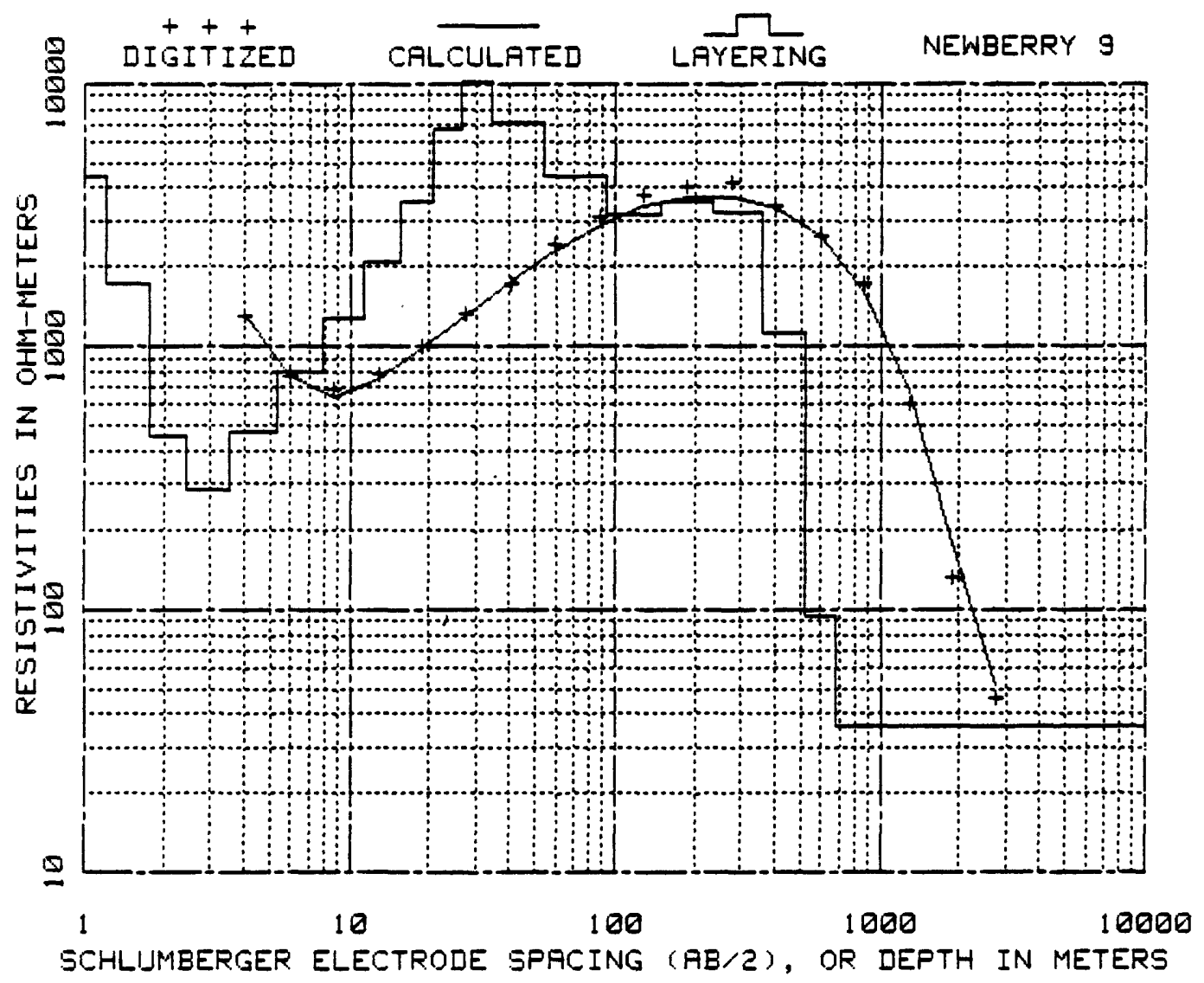

$\begin{array}{cr}\begin{array}{c}\text { INTERPRETED } \\ \text { DEPTH } \\ \text { IN METERS }\end{array} & \begin{array}{r}\text { INTERPRETED } \\ \text { RESISTIVI } \\ \text { IN OHM-METER }\end{array} \\ & \\ .13 & 3688.46 \\ .26 & 4347.84 \\ .39 & 3743.14 \\ .57 & 3352.98 \\ .33 & 4146.95 \\ 1.22 & 4441.19 \\ 1.76 & 1737.62 \\ 2.44 & 453.08 \\ 3.52 & 236.02 \\ 5.31 & 477.31 \\ 7.38 & 797.41 \\ 11.23 & 1271.35\end{array}$

$\begin{array}{cc}\text { INTERPRETED } & \text { INTERPRETED } \\ \text { DEPTH } & \text { RESISTIVITY } \\ \text { IN METERS } & \text { IN OHM-METERS }\end{array}$

$\begin{array}{rr}15.43 & 2076.00 \\ 20.59 & 3513.27 \\ 26.36 & 6710.40 \\ 34.35 & 10105.15 \\ 54.00 & 7147.16 \\ 92.33 & 4420.67 \\ 149.31 & 3169.34 \\ 233.20 & 3531.98 \\ 356.24 & 3236.24 \\ 522.77 & 1114.42 \\ 674.73 & 93.62 \\ 1000673.73 & 36.28\end{array}$




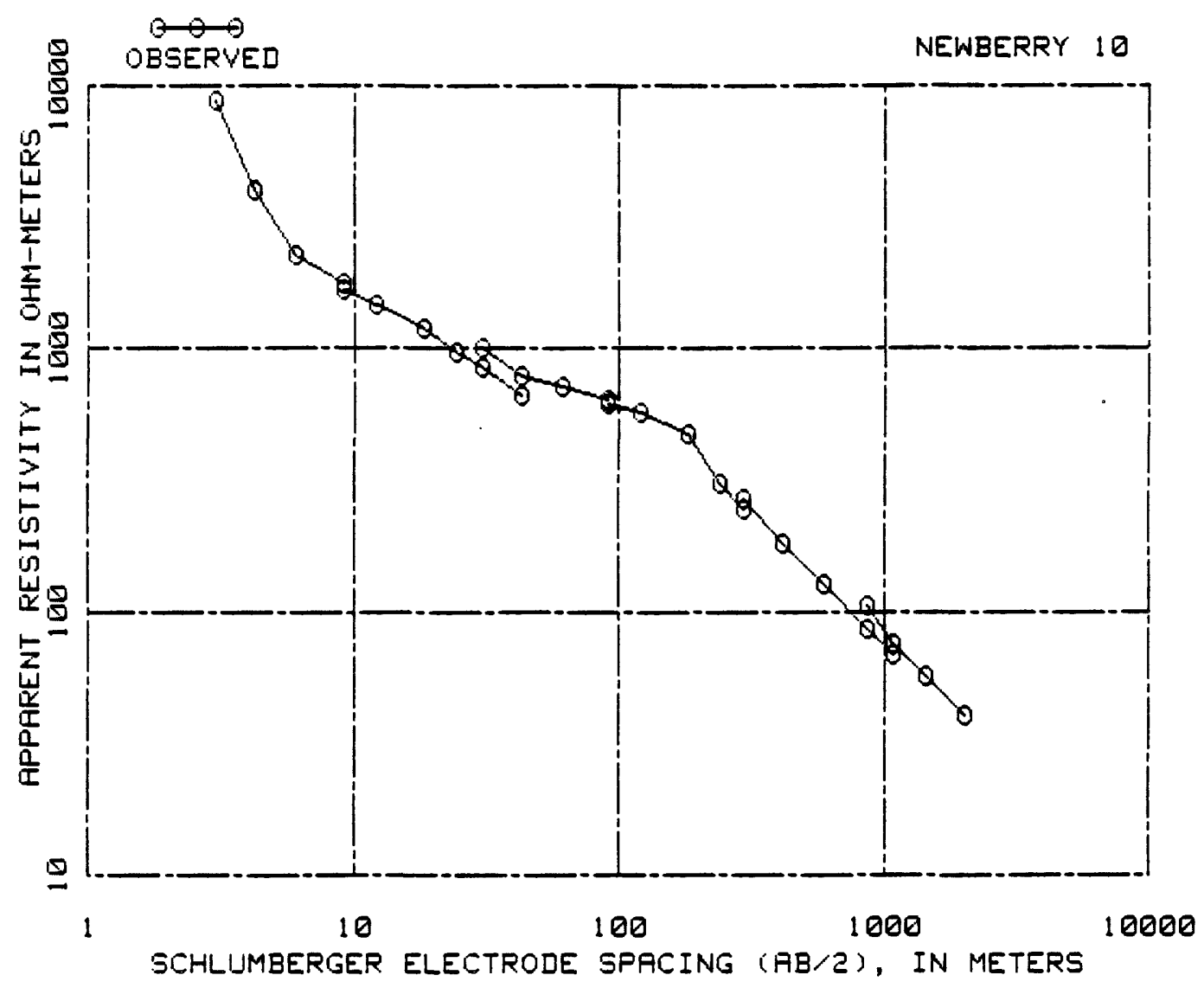

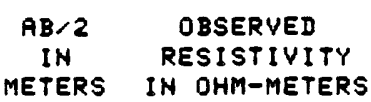

91.44

120.70

181.97

239.88

296.38

296.88

416.05

594.97

875.08

1094.84

875.08

1094.84

1448.41

2036.67
620.00

570.00

478.00

308.00

244.00

268.00

181.00

128.00

85.00

68.00

106.00

75.00

57.00

40.80 


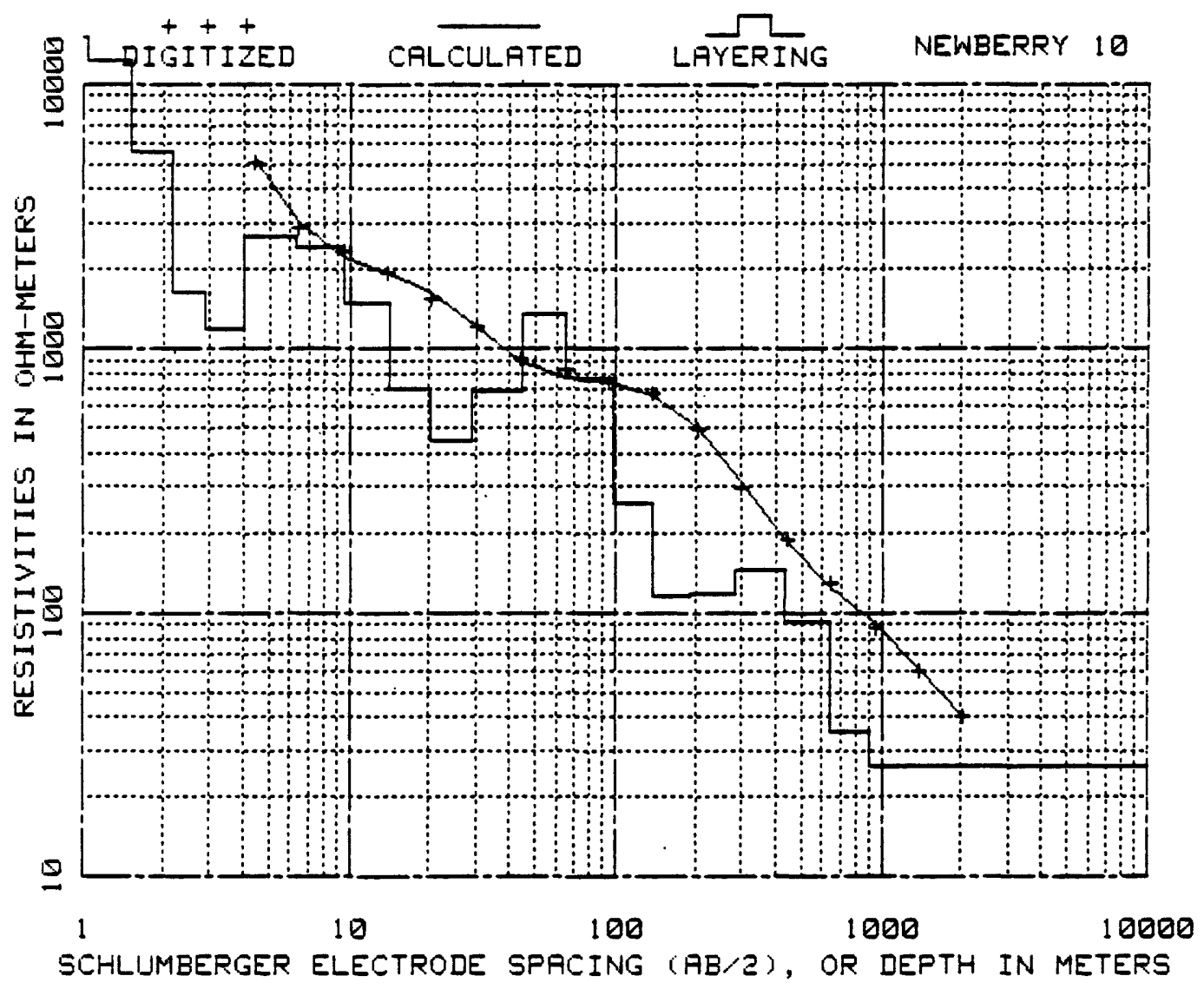

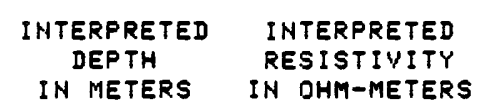

$\begin{array}{rr}.33 & 14422.22 \\ .48 & 14337.85 \\ 1.71 & 14774.02 \\ 1.52 & 15112.94 \\ 2.16 & 12142.13 \\ 2.86 & 5562.95 \\ 4.04 & 1650.62 \\ 5.29 & 1199.12 \\ 9.59 & 2650.42 \\ 14.23 & 2449.26 \\ & 1497.62\end{array}$
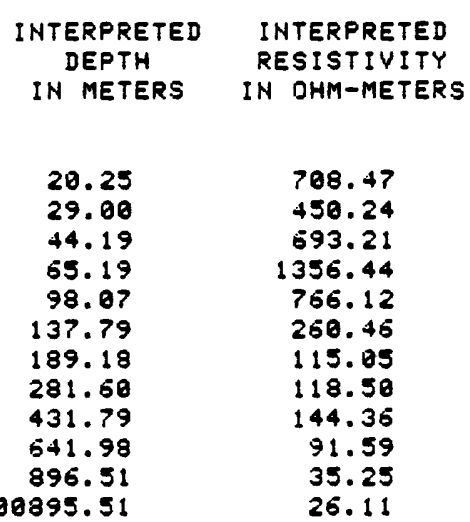

708.47

450.24

593.21

1356.44

766.12

260.46

115.05

118.50

144.36

91.59

35.25

26.11 


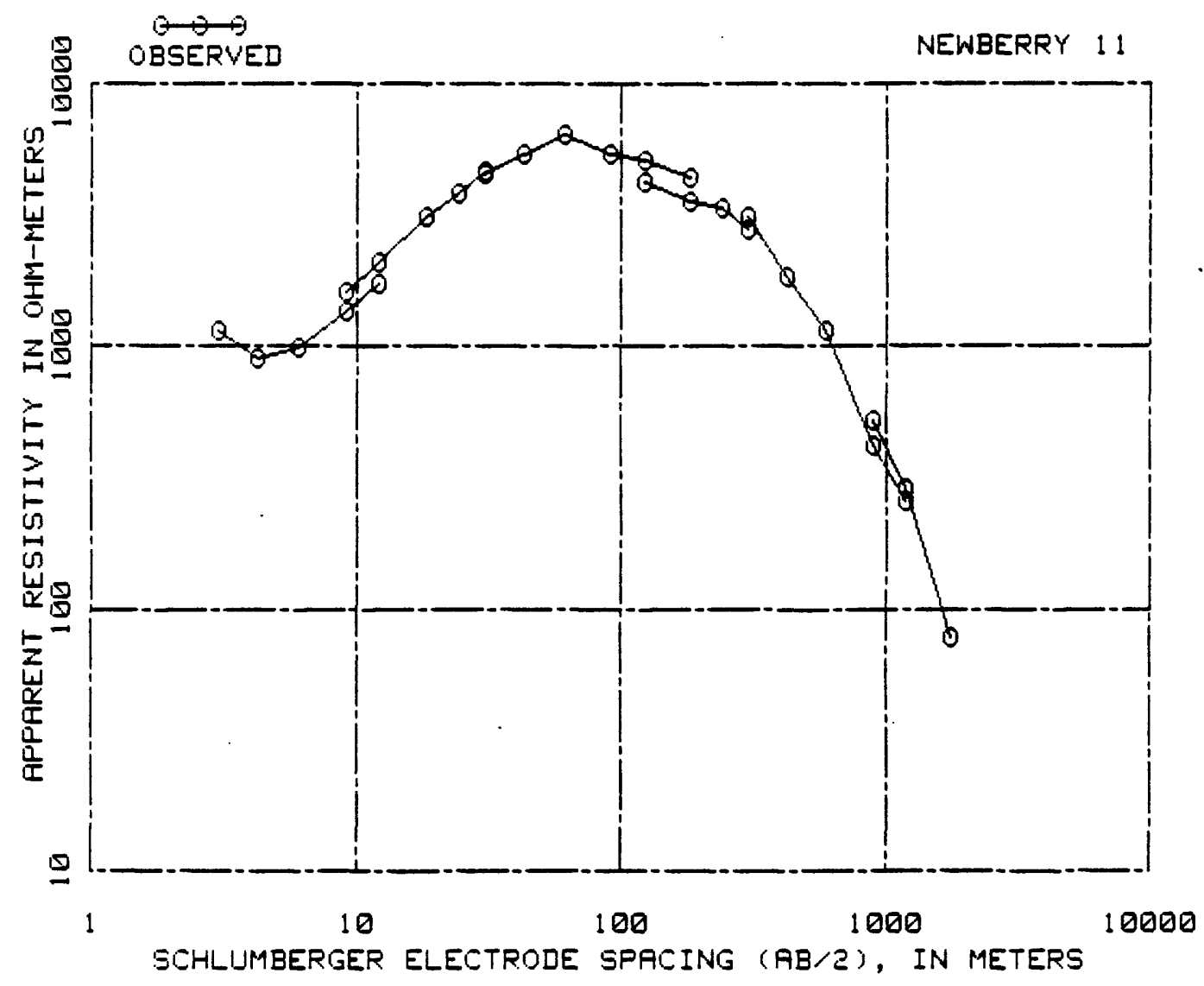

AB/2 OBSERVED

IN RESISTIVITY

METERS IN OHM-METERS

$\begin{array}{rr}3.05 & 1150.00 \\ 4.27 & 900.00 \\ 6.10 & 980.00 \\ 9.14 & 1350.00 \\ 12.19 & 1720.00 \\ 9.14 & 1600.00 \\ 12.19 & 2100.00 \\ 13.29 & 3080.00 \\ 24.38 & 3800.00 \\ 30.48 & 4600.00 \\ 30.48 & 4550.00 \\ 42.67 & 5350.00 \\ 60.76 & 6350.00 \\ 91.44 & 5400.00\end{array}$

$121.92 \quad 5100.00$

$182.88 \quad 4350.00$

$121.92 \quad 4200.00$

$182.88 \quad 3550.00$

$242.01 \quad 3364.00$

$302.06 \quad 2770.00$

$302.06 \quad 3079.00$

$422.15 \quad 1818.00$

$595.58 \quad 1137.00$

$898.25 \quad 416.00$

$1195.43 \quad 256.00$

$898.25 \quad 521.00$

$1195.43 \quad 286.00$

$1787.96 \quad 78.00$ 


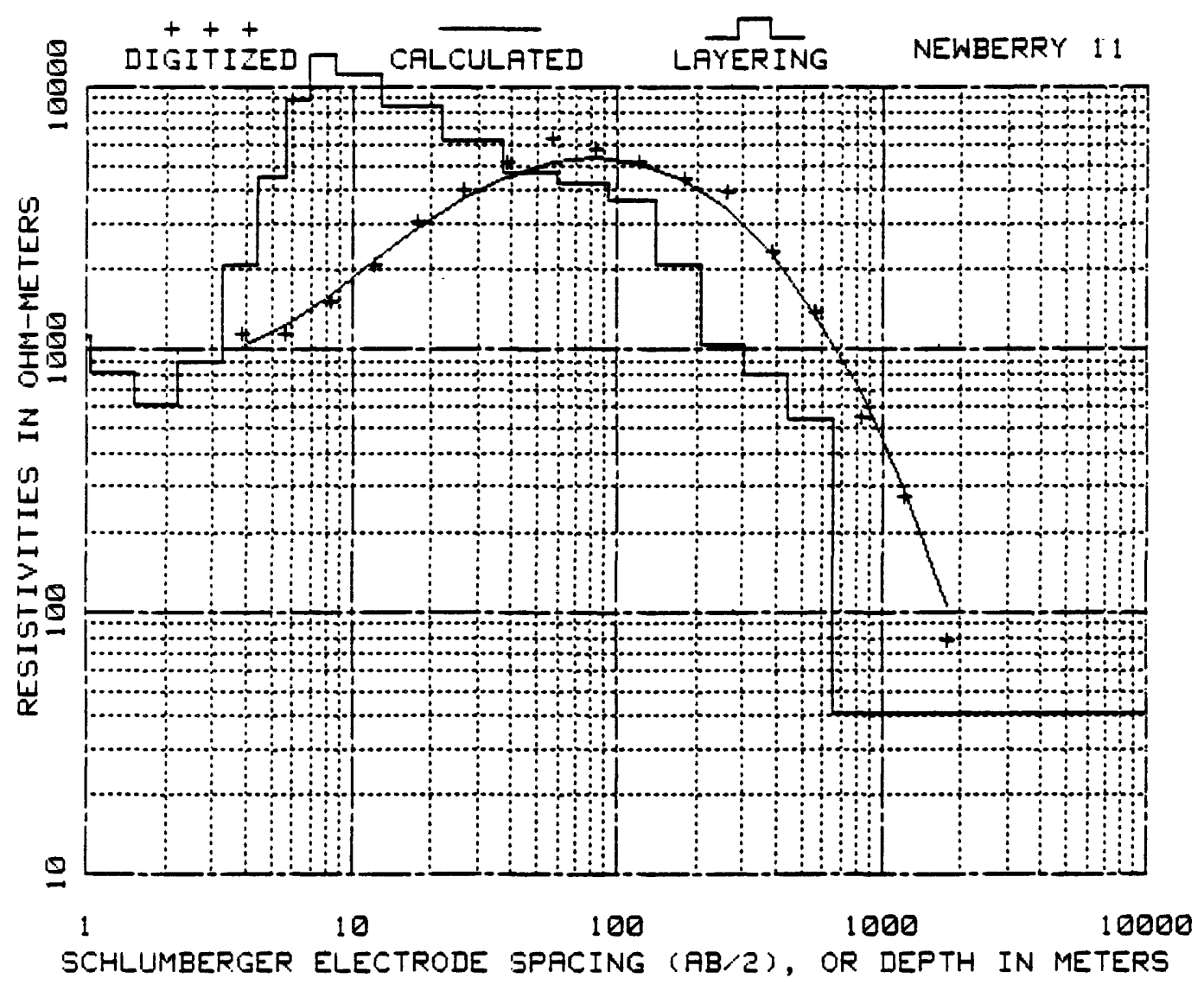

$\begin{array}{cc}\text { INTERPRETED } & \text { INTERPRETED } \\ \text { DEPTH } & \text { RESISTIVITY } \\ \text { IN METERS } & \text { IN OHM-METERS }\end{array}$

8.75

12.92

21.77

36.85

59.20

91.94

139.91

207.69

301.43

443.46

651.75

1000650.75
13297.78

11198.80

8406.87

6170.31

4693.02

4275.32

3783.04

2109.63

1048.29

799.57

544.83

41.06 


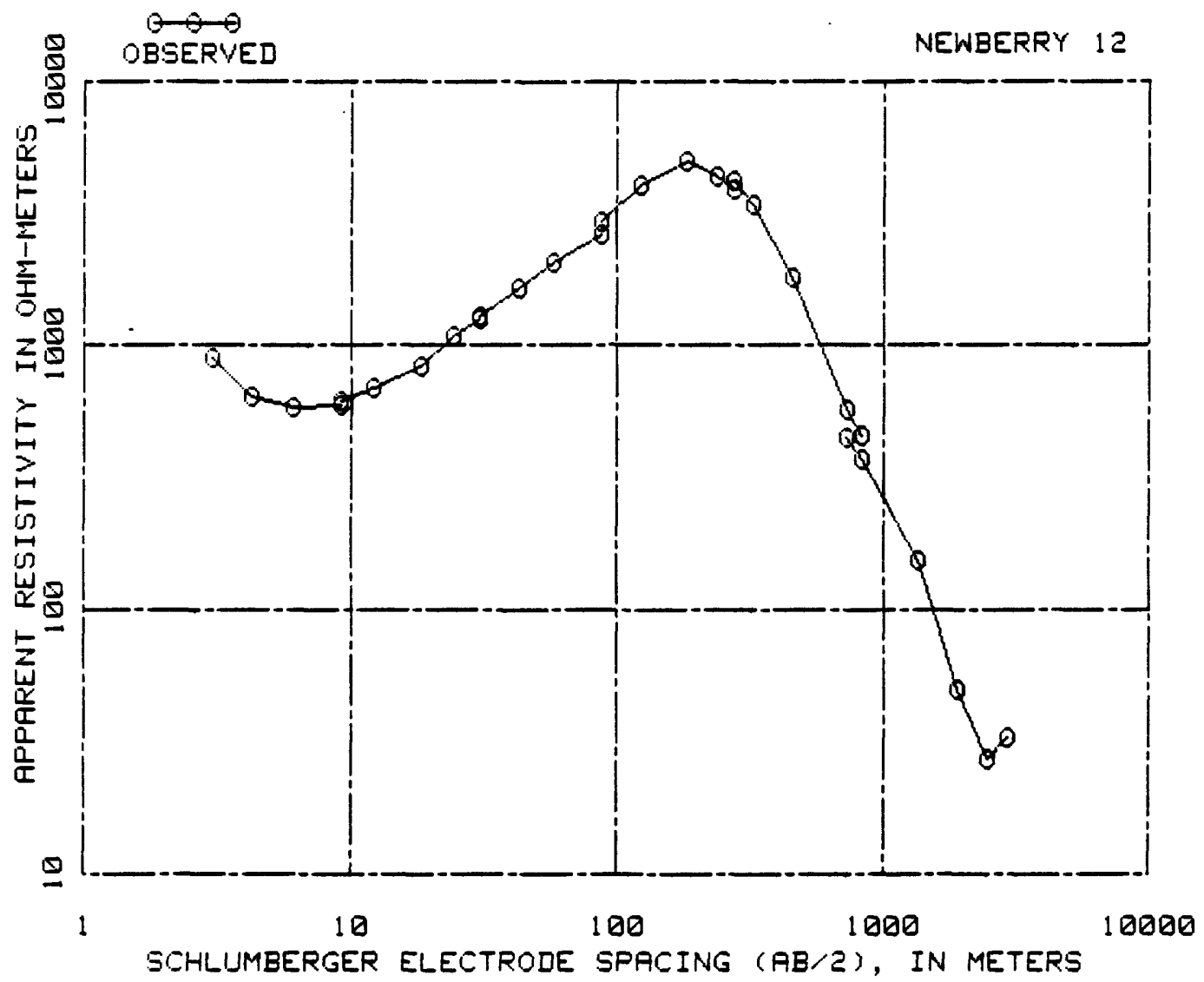

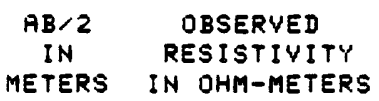

$\begin{array}{rr}3.05 & 900.00 \\ 4.27 & 645.00 \\ 6.10 & 580.00 \\ 9.14 & 590.00 \\ 9.14 & 620.00 \\ 12.19 & 695.00 \\ 18.29 & 840.00 \\ 24.38 & 1075.00 \\ 30.48 & 1250.00 \\ 30.48 & 1280.00 \\ 42.67 & 1650.00 \\ 58.52 & 2868.00 \\ 37.78 & 2609.00 \\ 87.78 & 2931.00\end{array}$

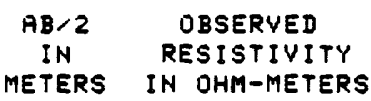

121.92

183.79

235.61

277.67

277.67

327.96

459.94

732.43

843.69

732.13

843.69

1357.58

1924.51

2491.74

2970.28
4037.00

4941.00

4351.00

3868.08

4208.00

3391.06

1783.00

576.08

455.00

445.08

372.00

153.08

50.00

27.08

33.80 


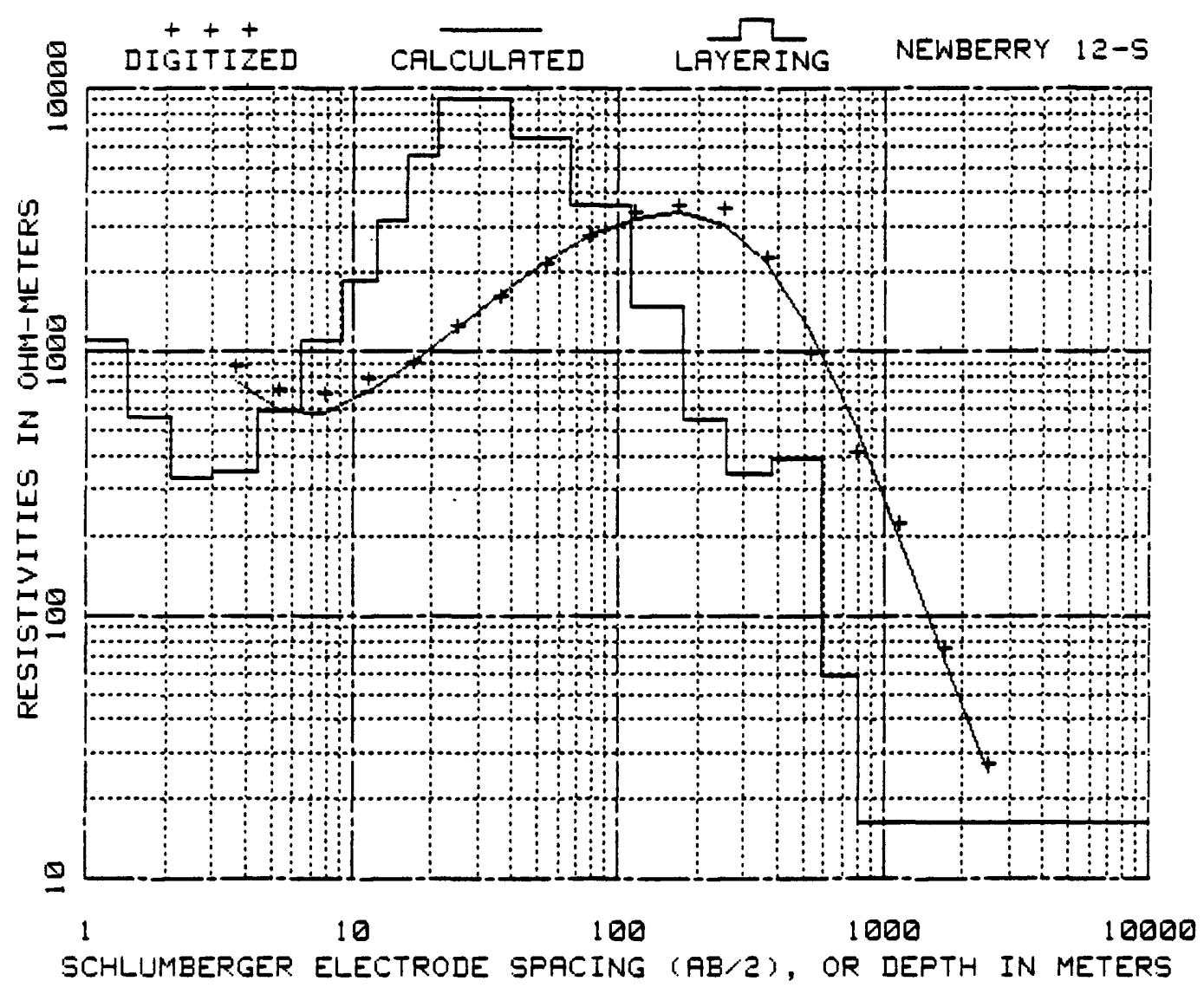

$\begin{array}{cc}\text { INTERPRETED } & \text { INTERPRETED } \\ \text { DEPTH } & \text { RESISTIYITY } \\ \text { IN METERS } & \text { IN DHM-METERS }\end{array}$

16.29
21.01
27.19
39.26
65.90
111.67
174.47
256.67
382.05
587.29
800.54
799.54

16.29

21. 01

39.26

65.90

11.67

38. 05

587.29

1000799.54
3149.66
5608.98
8976.98
8988.15
6428.37
3577.47
1493.44
554.96
341.42
392.41
59.24
16.27 


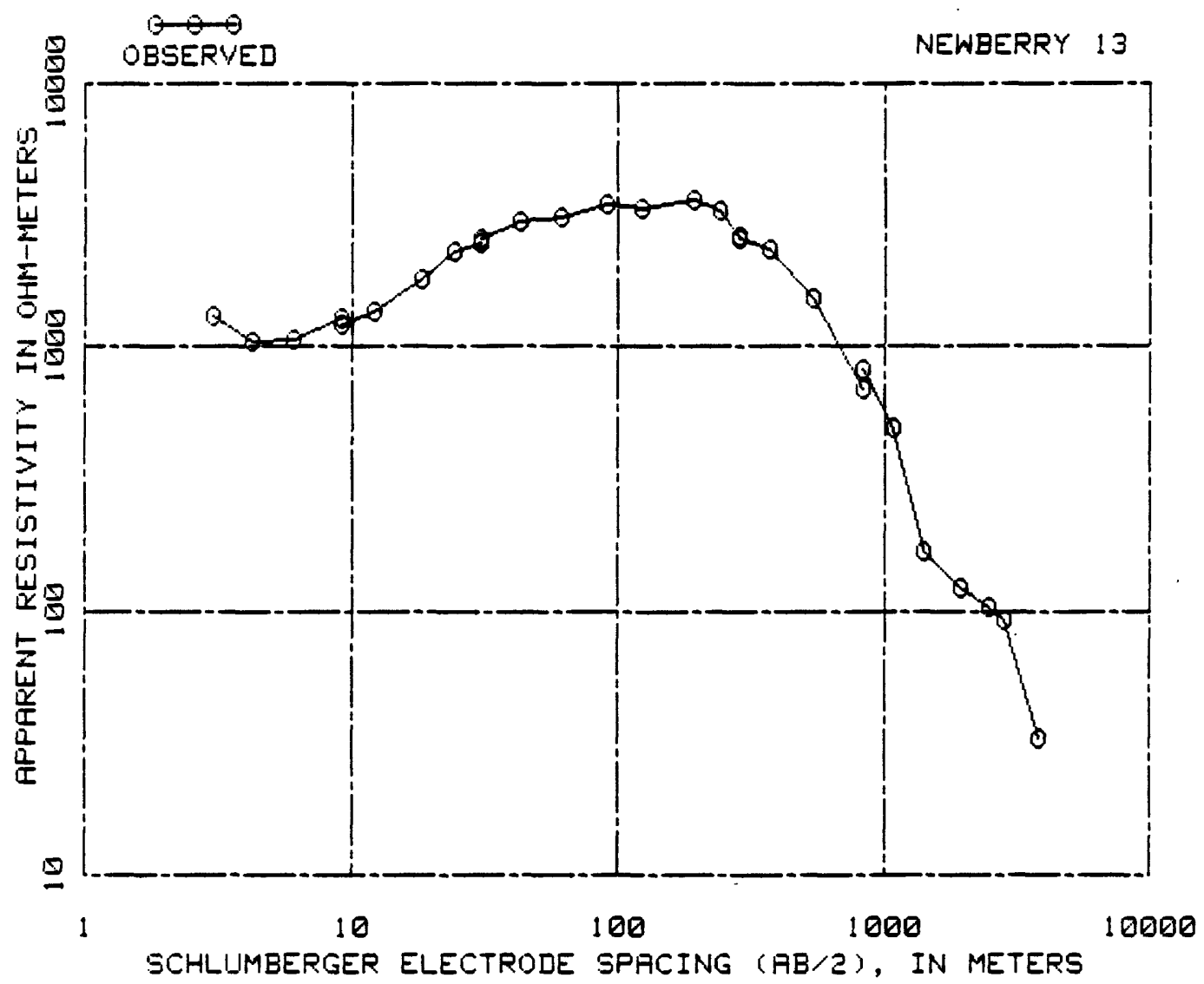

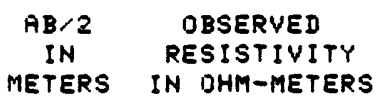

$\begin{array}{ll}3.05 & 1300.00 \\ 4.27 & 1050.00 \\ 6.10 & 1070.00 \\ 9.14 & 1275.00 \\ 9.14 & 1210.00 \\ 12.19 & 1350.00 \\ 18.29 & 1800.00 \\ 24.38 & 2280.00 \\ 30.48 & 2500.00 \\ 30.48 & 2550.00 \\ 42.57 & 3000.00 \\ 60.96 & 3125.00 \\ 91.44 & 3450.00 \\ 71.44 & 3500.00\end{array}$

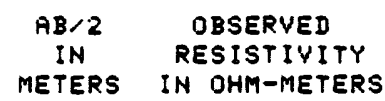

121.92

191.41

240.49

285. 70

285.90

375.51

541.63

837.29

837.29

1086.61

1417.32

1955.60

2505.15

2816.05

3831.95
3350.00

3579.00

3265.00

2634.00

2559.00

2332.00

1524.00

691.00

810.00

488.00

170.00

123.00

104.00

93.80

33.00 

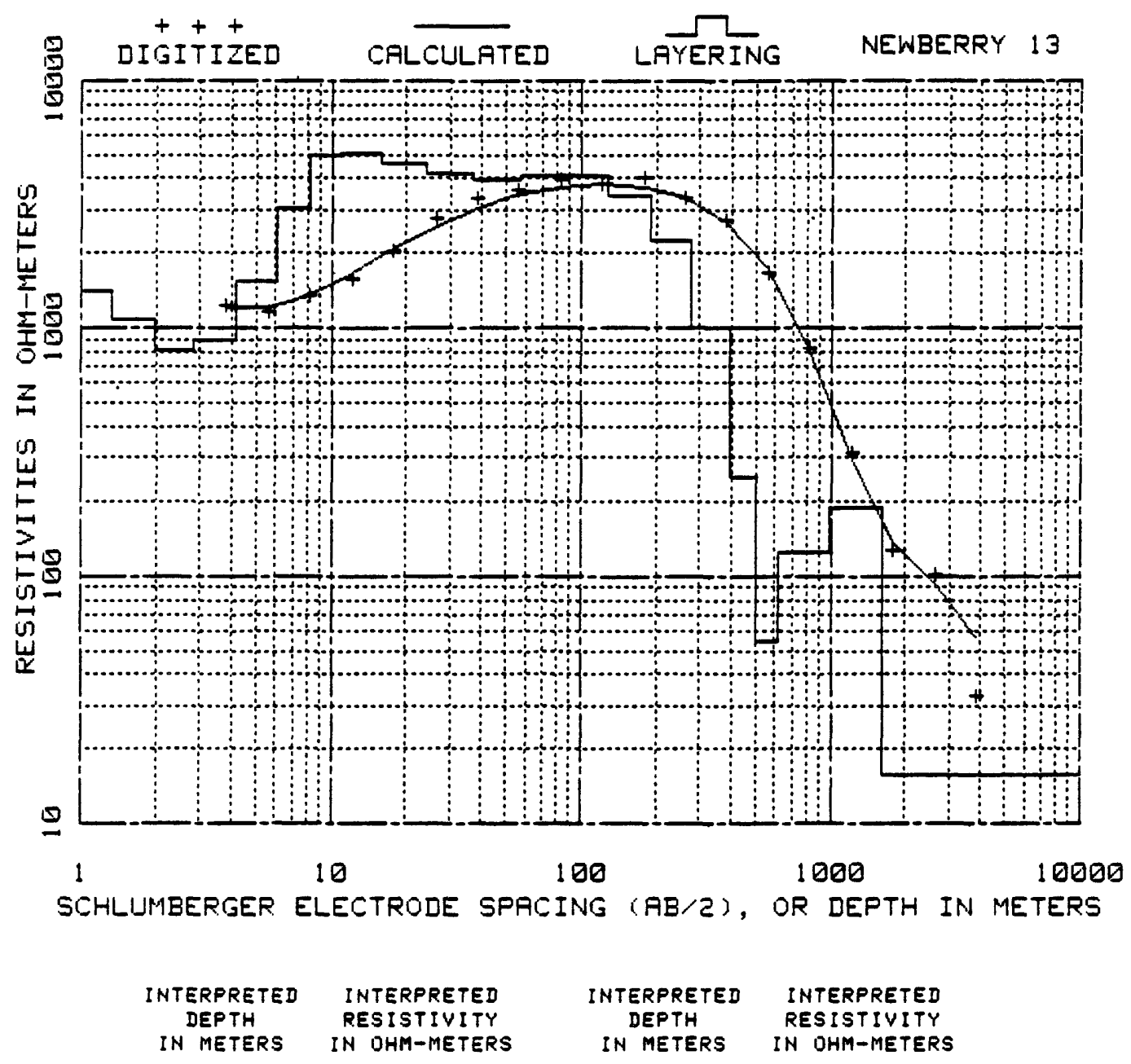

$\begin{array}{rr}.29 & 1503.68 \\ .42 & 1514.21 \\ .62 & 1534.23 \\ .91 & 1537.29 \\ 1.33 & 1410.12 \\ 1.75 & 1088.63 \\ 2.84 & 818.75 \\ 1.17 & 901.58 \\ 5.05 & 1543.99 \\ 8.21 & 3018.20 \\ 10.94 & 4949.15 \\ 15.71 & 5046.89\end{array}$

23.97
37.02
56.50
85.39
127.77
190.03
278.53
391.98
506.20
617.54
989.80
1019.13
1001609.13

4565.09
4171.68
3968.99
4079.56
4102.40
3433.59
2251.55
1004.70
252.30
54.61
124.15
190.30
15.68 


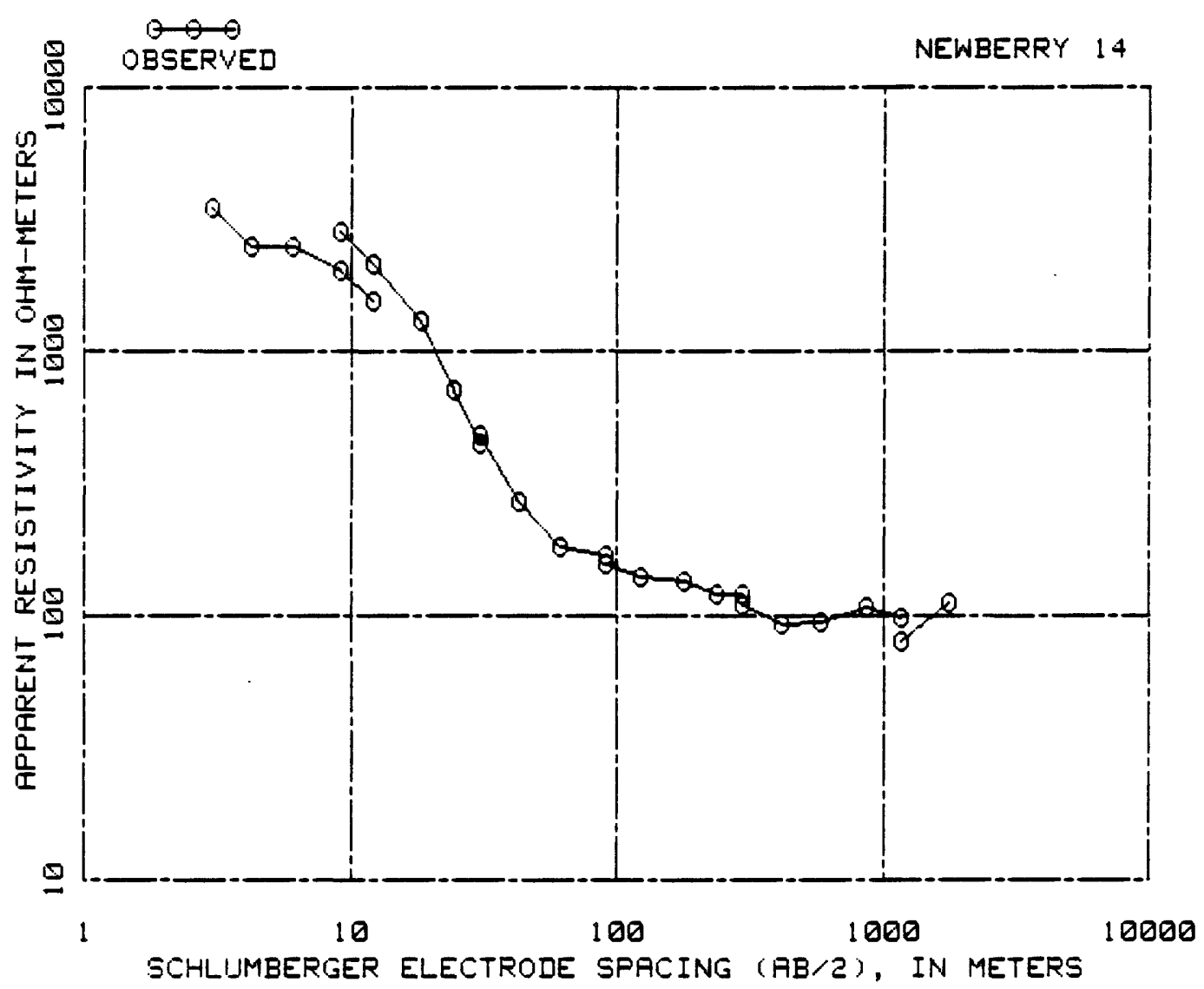

AB/2 OBSERVED

IN RESISTIVITY

METERS IN OHM-METERS

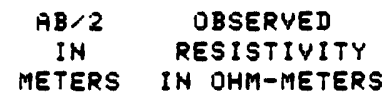

$\begin{array}{rr}3.05 & 3450.00 \\ 4.27 & 2500.00 \\ 5.10 & 2490.00 \\ 7.14 & 2000.00 \\ 12.19 & 1550.00 \\ 9.14 & 2800.00 \\ 12.19 & 2150.00 \\ 18.29 & 1300.00 \\ 24.38 & 710.00 \\ 30.48 & 450.00 \\ 30.48 & 480.00 \\ 42.67 & 270.00 \\ 50.96 & 180.00\end{array}$

$\begin{array}{rr}91 .+4 & 170.00 \\ 91.44 & 155.00 \\ 121.92 & 140.00 \\ 179.22 & 135.00 \\ 237.44 & 120.00 \\ 299.92 & 119.00 \\ 299.92 & 109.00 \\ 420.01 & 93.00 \\ 586.13 & 94.00 \\ 369.29 & 108.00 \\ 165.56 & 98.00 \\ 165.56 & 79.00 \\ 771.50 & 111.00\end{array}$



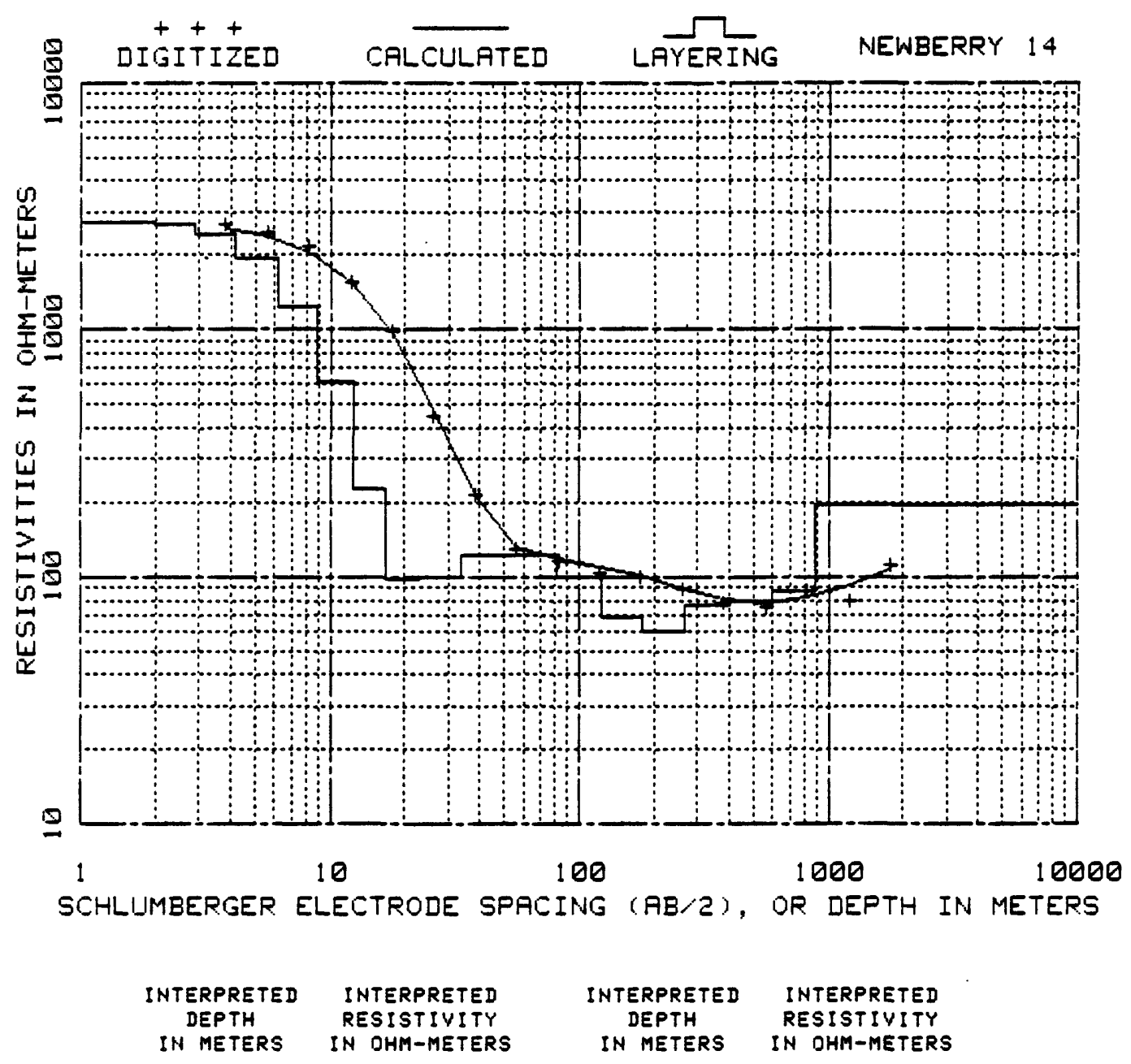

\begin{tabular}{|c|c|}
\hline $\begin{array}{r}.29 \\
.42 \\
.62 \\
.91 \\
1.33 \\
1.95 \\
3.86 \\
4.20 \\
5.14 \\
8.86 \\
12.44\end{array}$ & $\begin{array}{l}2716.14 \\
2714.63 \\
2713.88 \\
2715.77 \\
2726.69 \\
2717.80 \\
2663.67 \\
2448.12 \\
1932.96 \\
1227.16 \\
611.43\end{array}$ \\
\hline
\end{tabular}
16. 76
22.52
33.66
52.54
80.89
122.11
180.55
268.00
401.50
597.97
886.10

1000885.18

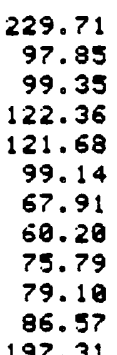




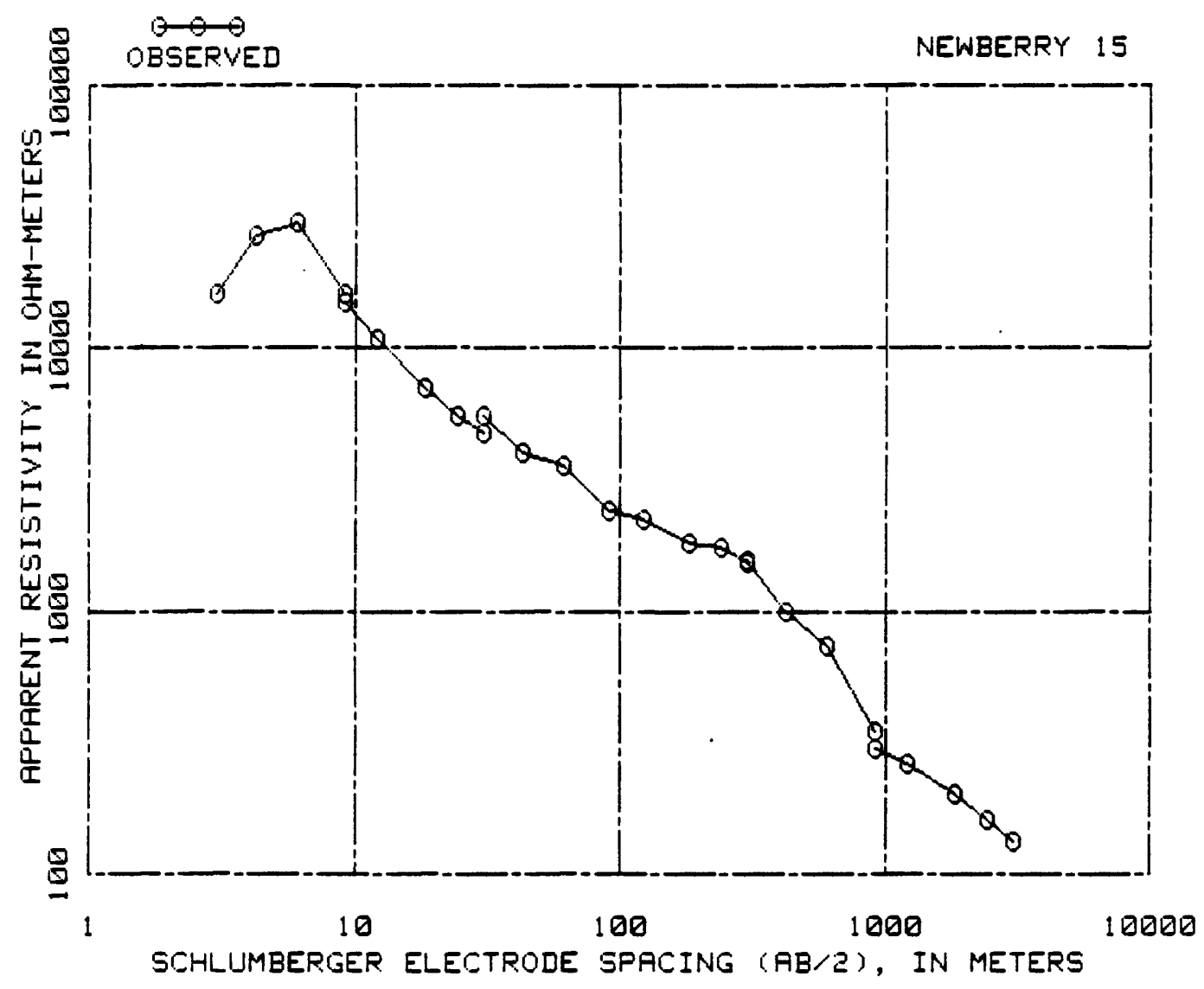

AB/2 OBSERYED

IN RESISTIVITY

METERS IN OHM-METERS

$\begin{array}{rr}3.05 & 16200.00 \\ 4.27 & 26800.00 \\ 6.10 & 30000.00 \\ 9.14 & 16000.00 \\ 9.14 & 14800.00 \\ 12.19 & 10800.00 \\ 18.29 & 7000.00 \\ 34.38 & 5500.00 \\ 30.48 & 4700.00 \\ 30.48 & 5500.00 \\ 42.67 & 4000.00 \\ 50.96 & 3600.00 \\ 91.44 & 2400.00\end{array}$

$\begin{array}{rr}91.44 & 2400.00 \\ 121.92 & 2250.00 \\ 182.88 & 1825.00 \\ 243.84 & 1750.00 \\ 304.80 & 1540.00 \\ 304.80 & 1550.00 \\ 426.72 & 1000.00 \\ 609.60 & 740.00 \\ 914.40 & 350.00 \\ 914.40 & 300.00 \\ 1219.20 & 260.00 \\ 1828.80 & 200.00 \\ 2438.40 & 160.00 \\ 3048.00 & 133.00\end{array}$



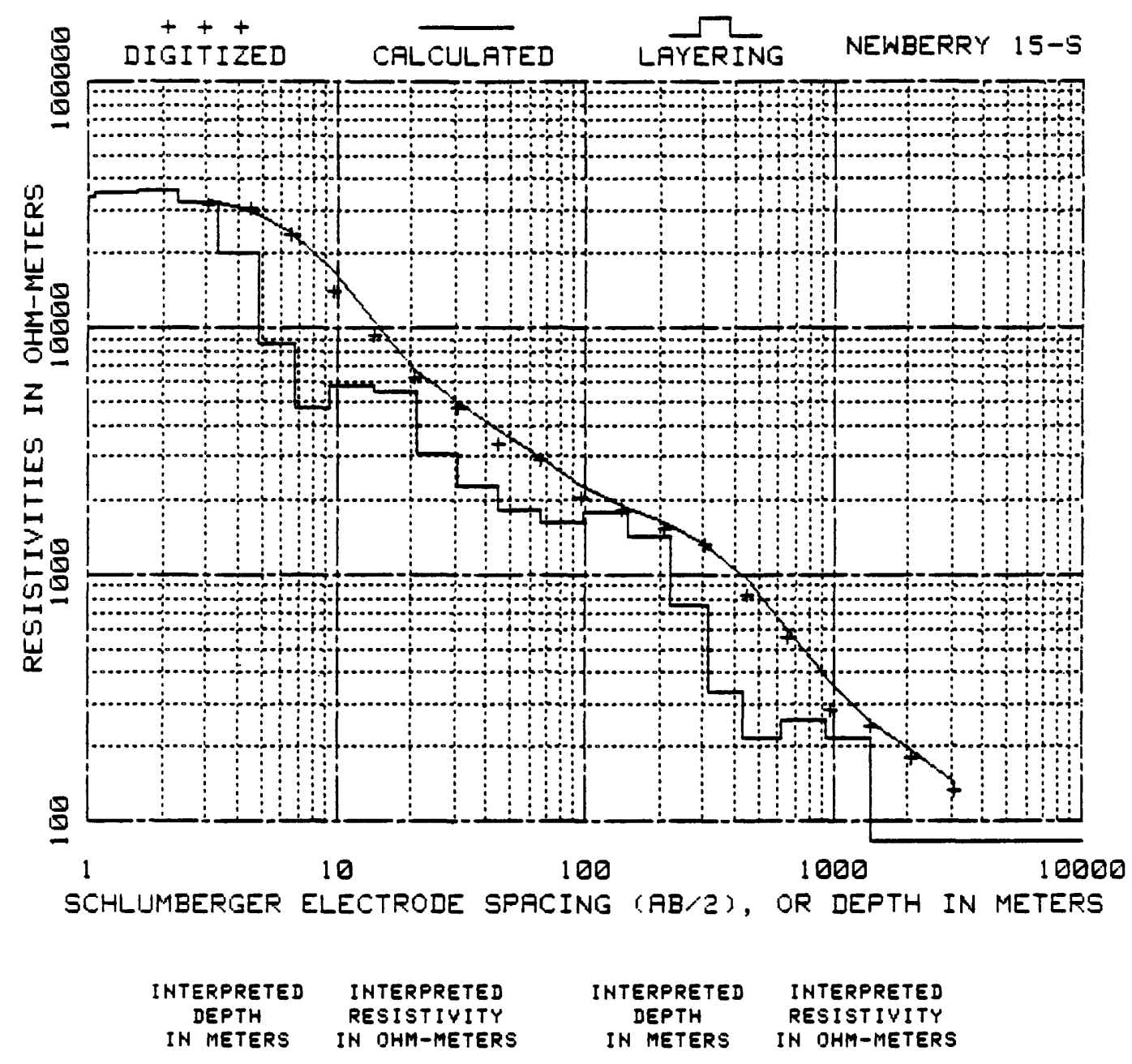

$\begin{array}{cc}\text { INTERPRETED } & \text { INTERPRETED } \\ \text { DEPTH } & \text { RESISTIVITY } \\ \text { IN METERS } & \text { IN DHM-METERS }\end{array}$

$\begin{array}{rr}.23 & 34408.04 \\ .34 & 34395.33 \\ .49 & 34315.36 \\ .72 & 34207.03 \\ 1.06 & 34380.11 \\ 1.56 & 35256.77 \\ 2.28 & 36307.46 \\ 3.35 & 32445.85 \\ 4.86 & 20025.03 \\ 5.78 & 8719.20 \\ 9.44 & 4729.45 \\ 14.12 & 5806.73\end{array}$

$\begin{array}{rr}21.18 & 5504.01 \\ 30.78 & 3061.90 \\ 44.98 & 2285.26 \\ 66.19 & 1815.46 \\ 98.24 & 1608.63 \\ 147.29 & 1797.05 \\ 218.36 & 1430.60 \\ 313.61 & 745.16 \\ 435.33 & 333.58 \\ 519.01 & 215.36 \\ 938.48 & 259.42 \\ 1411.60 & 218.24 \\ 1001410.60 & 83.50\end{array}$




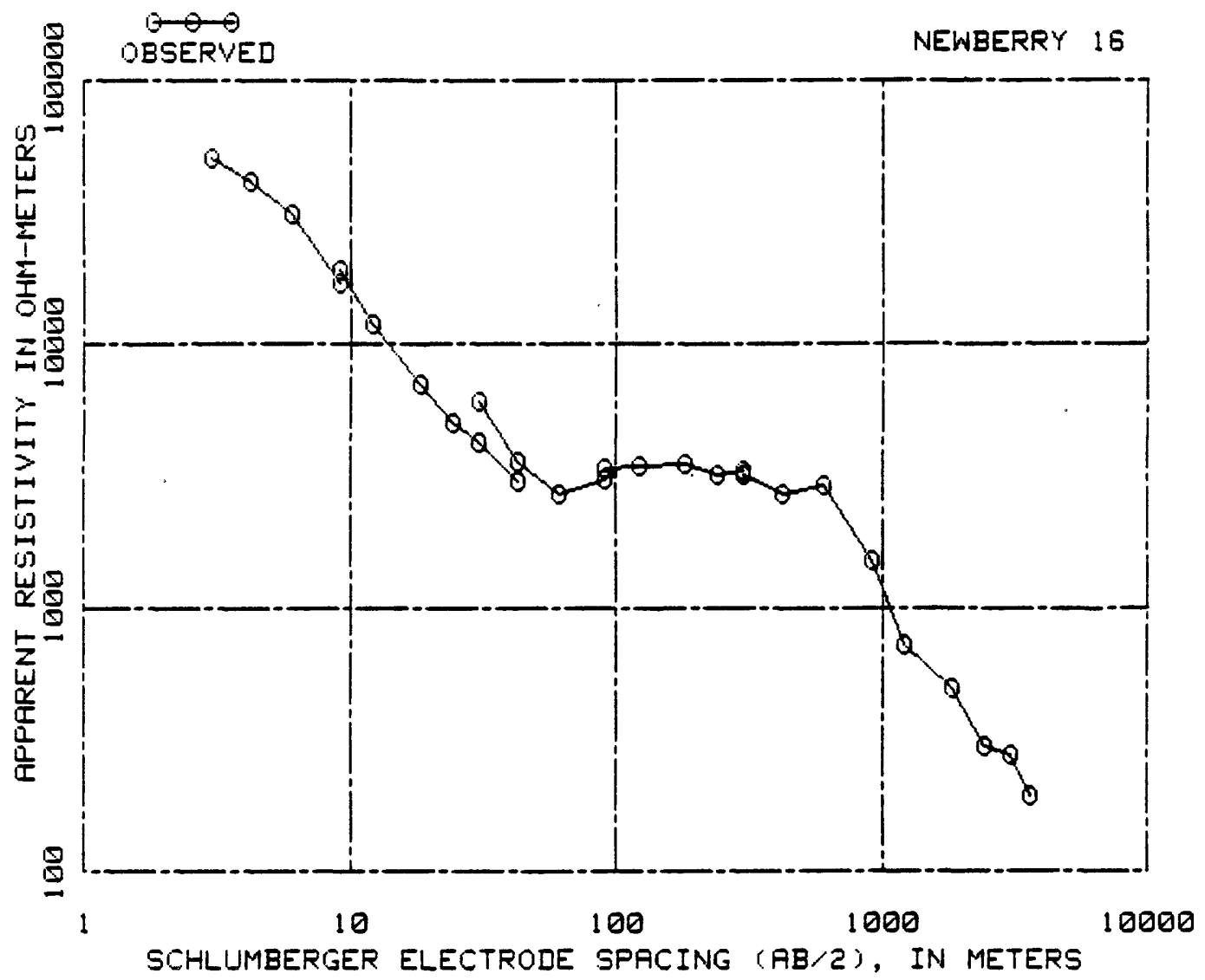

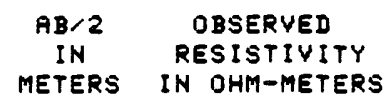

91.44

121.92

182.88

243.84

304.80

304.80

426.72

609.60

914.40

914.40

1219.20

1828.80

2438.40

3048.00

3657.60
3400.00

3440.00

3500.00

3200.00

3300.00

3200.00

2700.00

2900.00

1500.00

1500.00

725.00

500.00

300.00

278.00

194.00 


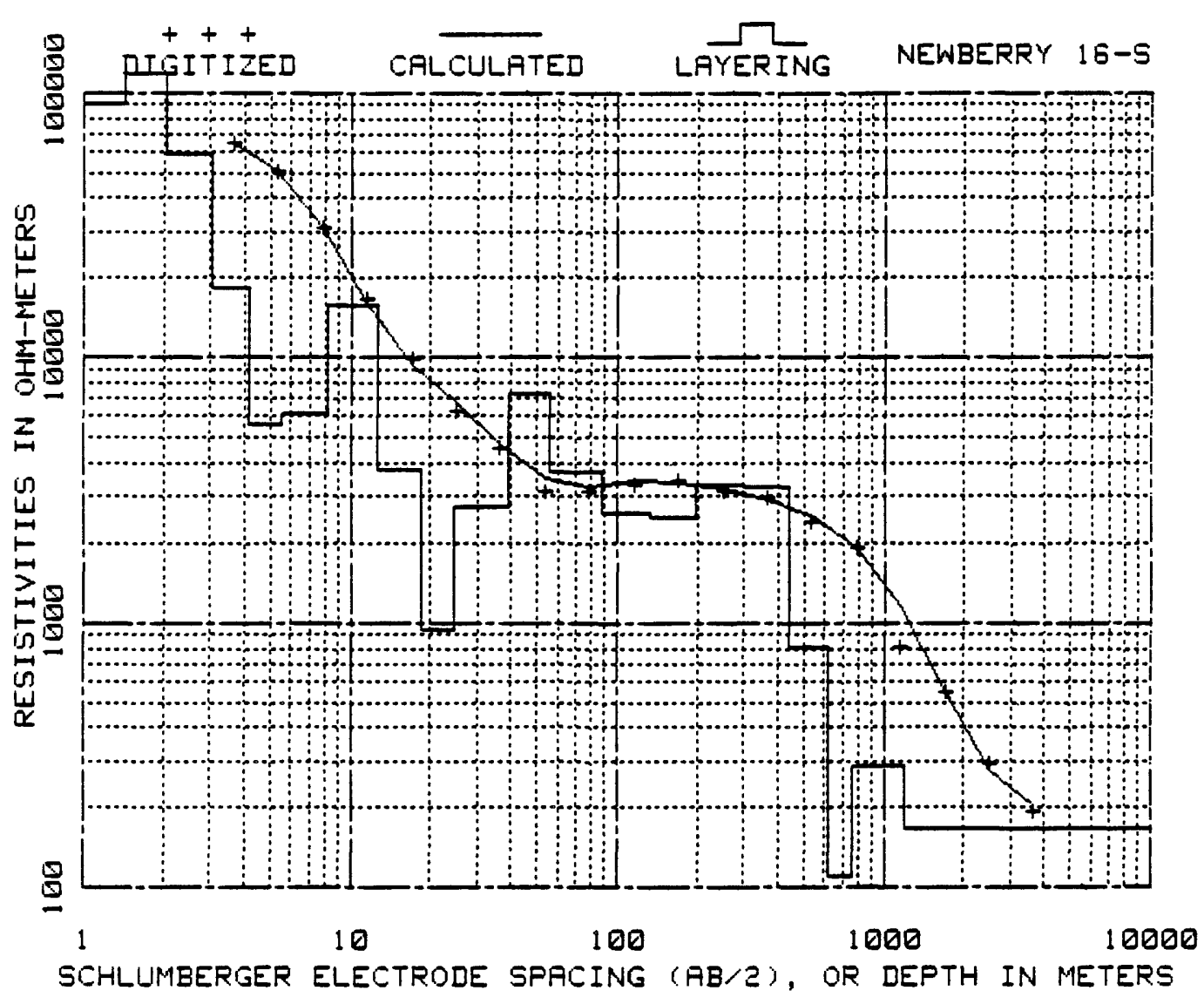

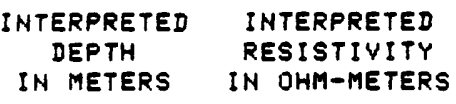

18.43
24.50
38.92
55.84
87.02
131.94
198.46
296.91
441.77
615.25
755.92
186.67
185.67
3788.67

938.82

2761.78

7302.57

3698.37

2586.32

2487.56

3330.28

3227.93

806.77

109.35

285.90

166.76 


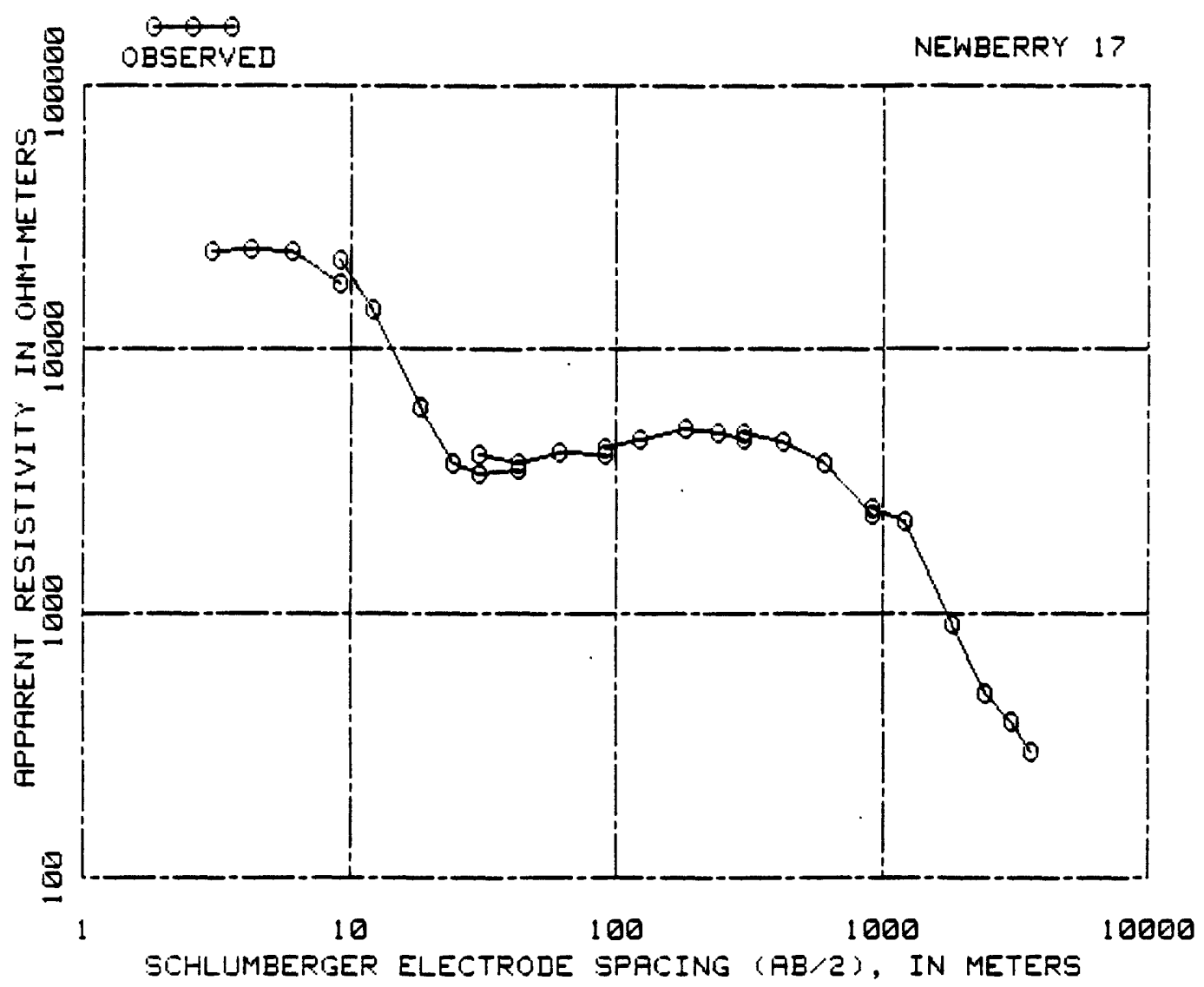

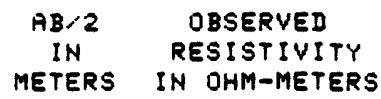

$\begin{array}{rr}91.44 & 4200.00 \\ 121.92 & 4550.00 \\ 182.88 & 5000.00 \\ 243.84 & 4800.00 \\ 304.30 & 4600.00 \\ 304.30 & 4800.00 \\ 426.72 & 4500.00 \\ 609.60 & 3700.00 \\ 914.40 & 2350.00 \\ 914.40 & 2500.00 \\ 219.20 & 2250.00 \\ 828.80 & 900.00 \\ 2438.40 & 500.00 \\ 3048.00 & 390.00 \\ 3657.60 & 300.00\end{array}$




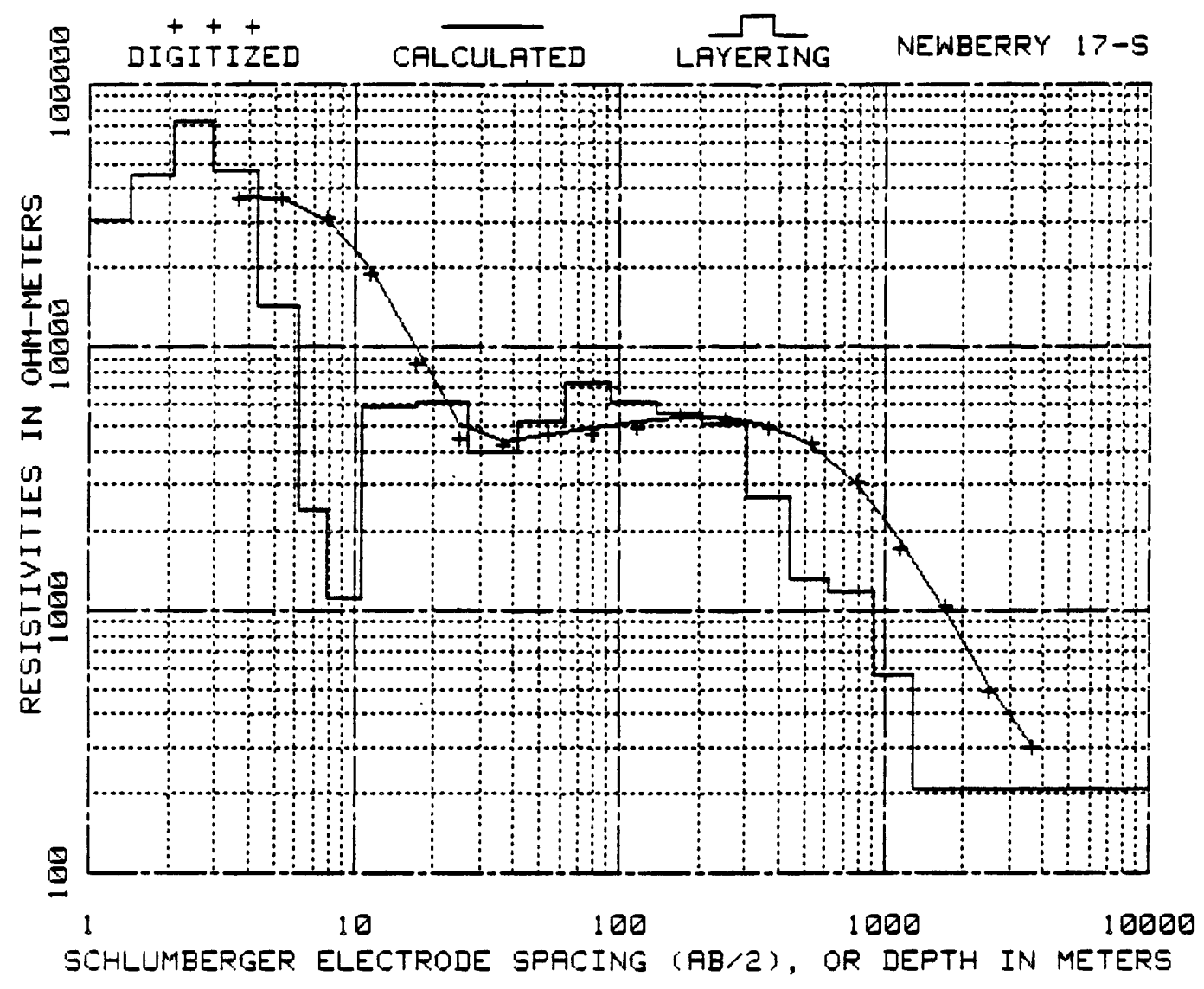

$\begin{array}{cc}\text { INTERPRETED } & \text { INTERPRETED } \\ \text { DEPTH } & \text { RESISTIVITY } \\ \text { IN METERS } & \text { IN OHM-METERS }\end{array}$

17.08

26.71

41.09

62.28

91.89

137.53

204.78

303.31

440.01

623.22

912.27

1297.26

1001296.26
5924.39

6199.01

3996.90

5248.35

7254.29

6144.44

5631.37

5098.46

2693. 02

1325.26

1186.54

567.95

209.91 


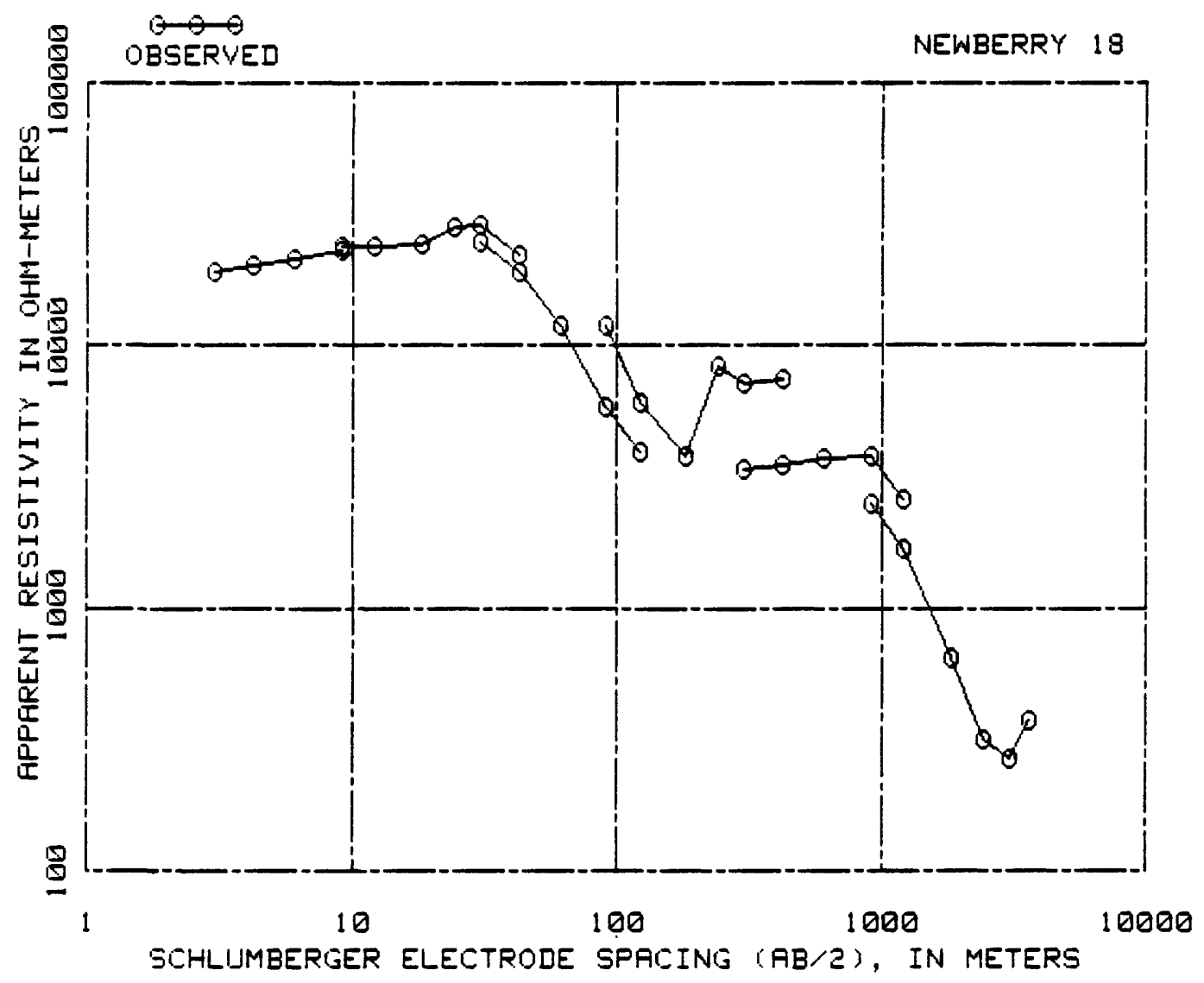

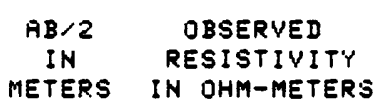

$\begin{array}{rr}3.05 & 18900.00 \\ +.27 & 20000.00 \\ 6.10 & 21250.00 \\ 9.14 & 23000.00 \\ 9.14 & 24000.00 \\ 12.19 & 23950.00 \\ 18.29 & 24300.00 \\ 24.38 & 28000.00 \\ 30.48 & 29000.00 \\ +2.67 & 22000.00 \\ 30.48 & 25000.00 \\ +2.67 & 19000.00 \\ 50.96 & 12000.00 \\ 91.44 & 5800.00 \\ 21.32 & 3960.00 \\ 91.44 & 12000.00\end{array}$

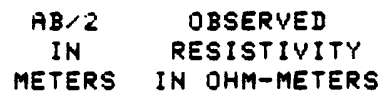

$\begin{array}{rr}121.92 & 6000.00 \\ 182.88 & 3800.00 \\ 243.84 & 8360.00 \\ 304.80 & 7200.00 \\ 426.72 & 7500.00 \\ 304.80 & 3400.00 \\ 426.72 & 3500.00 \\ 609.60 & 3700.00 \\ 914.40 & 3750.00 \\ 1213.20 & 2600.00 \\ 914.40 & 2500.00 \\ 1219.20 & 1790.00 \\ 1828.80 & 650.00 \\ 2438.40 & 317.00 \\ 3048.00 & 265.00 \\ 3657.60 & 378.00\end{array}$




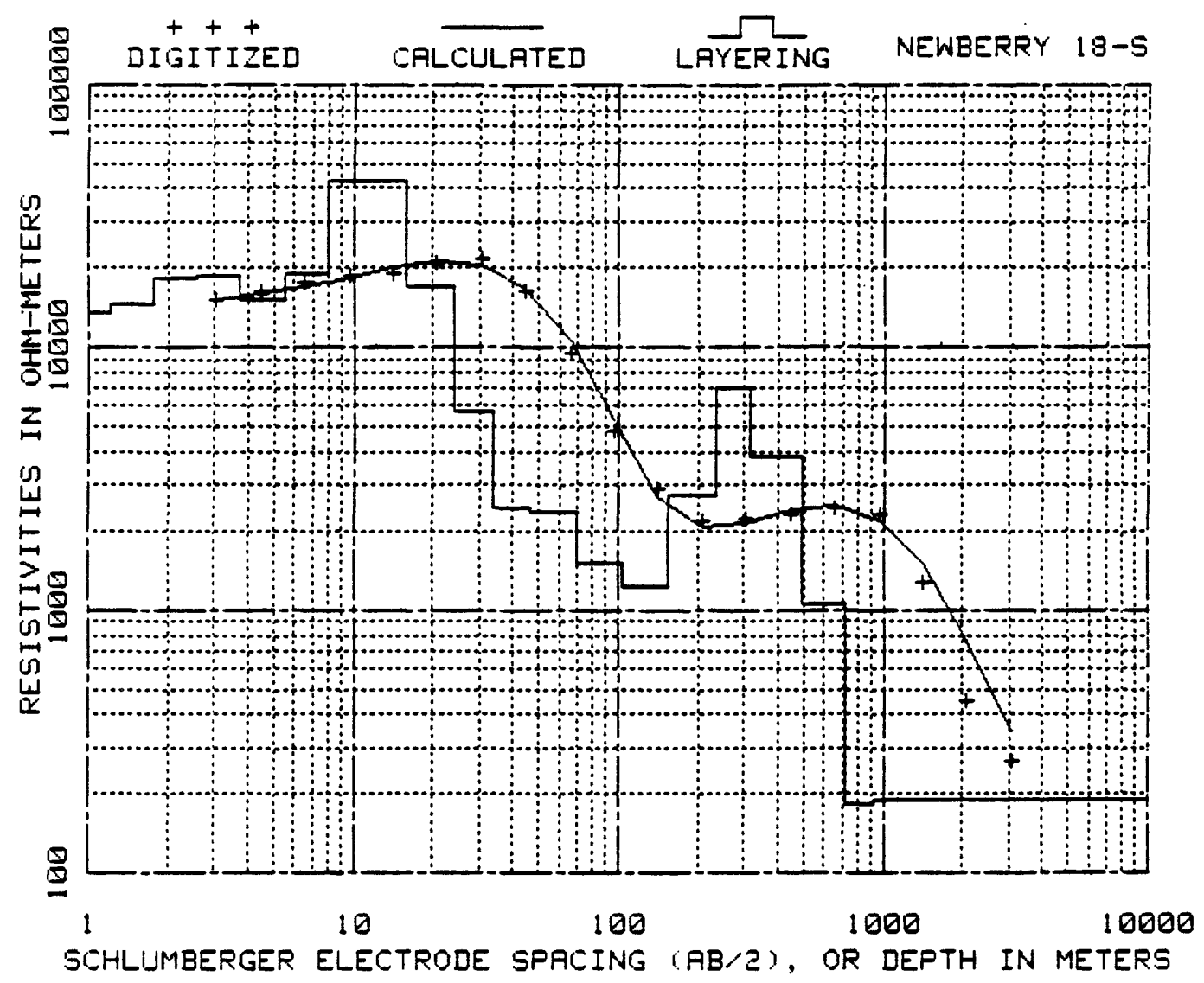

$\begin{array}{cc}\begin{array}{c}\text { INTERPRETED } \\ \text { DEPTH }\end{array} & \begin{array}{c}\text { INTERPRET } \\ \text { RESISTIVI } \\ \text { IN METERS }\end{array} \\ \text { IN OHM-MET } \\ \\ .18 & 14068.13 \\ .26 & 13976.51 \\ .38 & 14891.76 \\ .56 & 14271.37 \\ .82 & 14016.83 \\ 1.28 & 13462.42 \\ 1.75 & 14579.62 \\ 2.56 & 18172.98 \\ 3.75 & 18749.68 \\ 5.52 & 15334.72 \\ 8.10 & 18940.13 \\ 10.98 & 42626.45\end{array}$

$\begin{array}{cc}\text { INTERPRETED } & \text { INTERPRETED } \\ \text { DEPTH } & \text { RESISTIVITY } \\ \text { IN METERS } & \text { IN OHM-METERS }\end{array}$

$\begin{array}{rr}15.80 & 42427.81 \\ 23.85 & 17096.06 \\ 33.65 & 5699.64 \\ 46.70 & 2439.23 \\ 69.73 & 2372.63 \\ 103.12 & 1514.37 \\ 154.36 & 1223.85 \\ 234.93 & 2751.76 \\ 315.90 & 7054.45 \\ 489.99 & 3887.11 \\ 714.60 & 1047.63 \\ 921.48 & 183.27 \\ 1009920.48 & 191.71\end{array}$




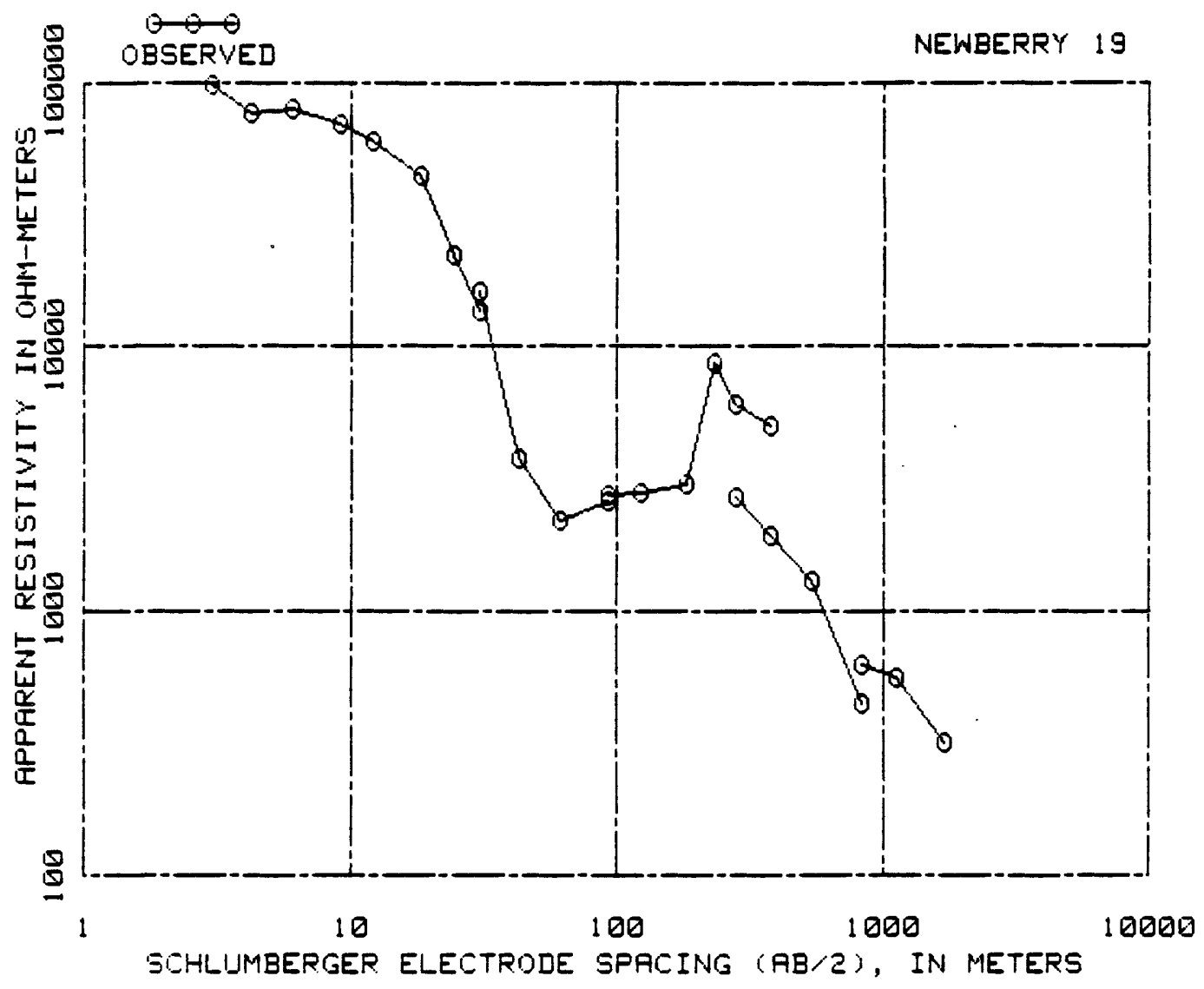

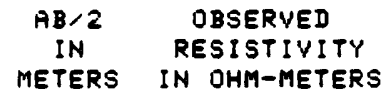

93.57

123.14

184.10

231.95

281.34

380.70

281.94

380.70

542.24

835.15

835.15

1136.29

1695.60

$$
\begin{aligned}
& 2721.00 \\
& 2781.00 \\
& 3034.00 \\
& 3627.00 \\
& 5998.00 \\
& 4967.00 \\
& 2695.00 \\
& 1915.00 \\
& 1305.00 \\
& 445.00 \\
& 626.00 \\
& 558.00 \\
& 319.00
\end{aligned}
$$




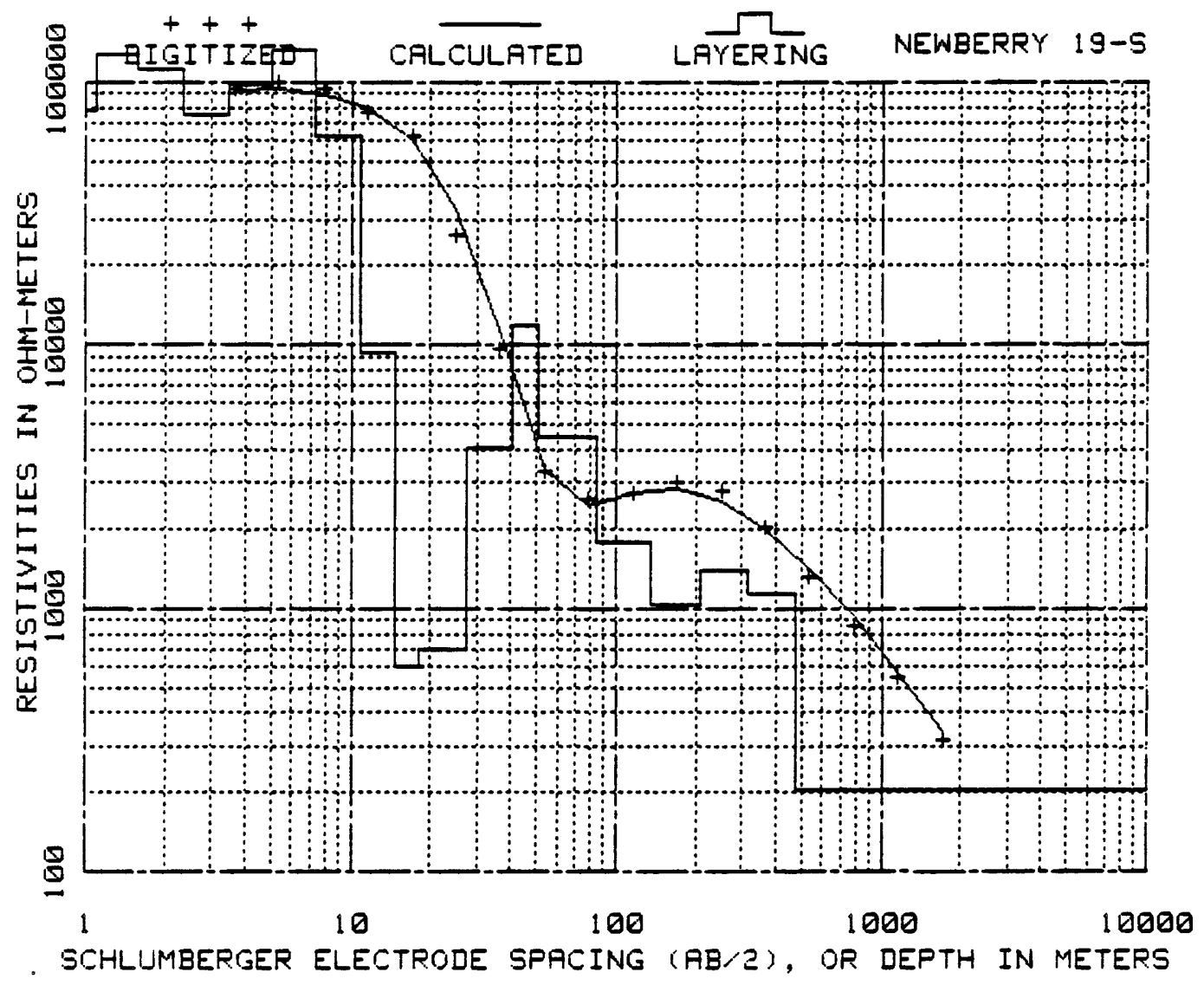

\begin{tabular}{|c|c|}
\hline $\begin{array}{c}\text { INTERPRETED } \\
\text { DEPTH }\end{array}$ & $\begin{array}{l}\text { INTERPRET } \\
\text { RESISTIVI }\end{array}$ \\
\hline IN METERS & IN DHM-MET \\
\hline $\begin{array}{r}.16 \\
.24 \\
.35 \\
.51 \\
.75 \\
1.10 \\
1.58 \\
2.33 \\
3.44 \\
5.06 \\
7.36\end{array}$ & $\begin{array}{r}88983.82 \\
82316.62 \\
33336.18 \\
85708.00 \\
67852.16 \\
78228.39 \\
127868.82 \\
111534.94 \\
75661.53 \\
38318.42 \\
132667.84\end{array}$ \\
\hline
\end{tabular}

$\begin{array}{cc}\text { INTERPRETED } & \text { INTERPRETED } \\ \text { DEPTH } & \text { RESISTIVITY } \\ \text { IN METERS } & \text { IN UHM-METERS }\end{array}$

10.82
14.66
17.98
27.21
40.32
50.79
84.74
134.79
206.28
316.94
478.59
477.59

62273.88
9277.80
601.27
691.52
4060.63
11903.71
4436.66
1775.16
1039.60
1406.38
1130.99
203.39 


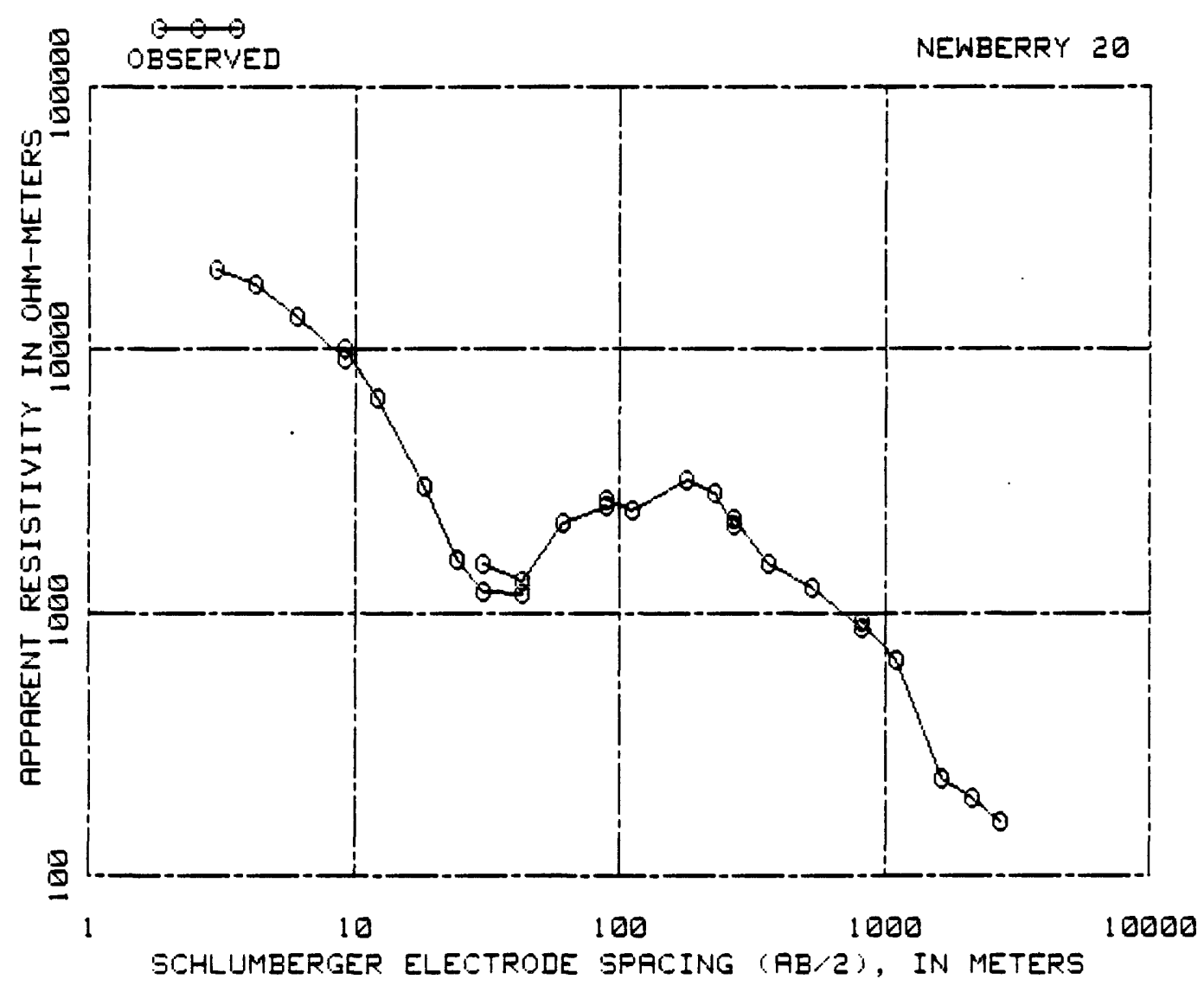

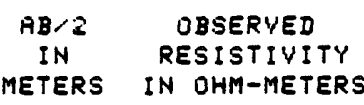

88.70

111.25

179.83

228.90

270.97

270.97

366.06

535.23

822.05

822.05

1118.92

1650.49

2125.98

2725.22
2679.00

2458.00

3205.00

2829.00

2171.00

2282.00

1521.00

1240.00

872.00

929.00

654.00

234.00

198.00

162.00 


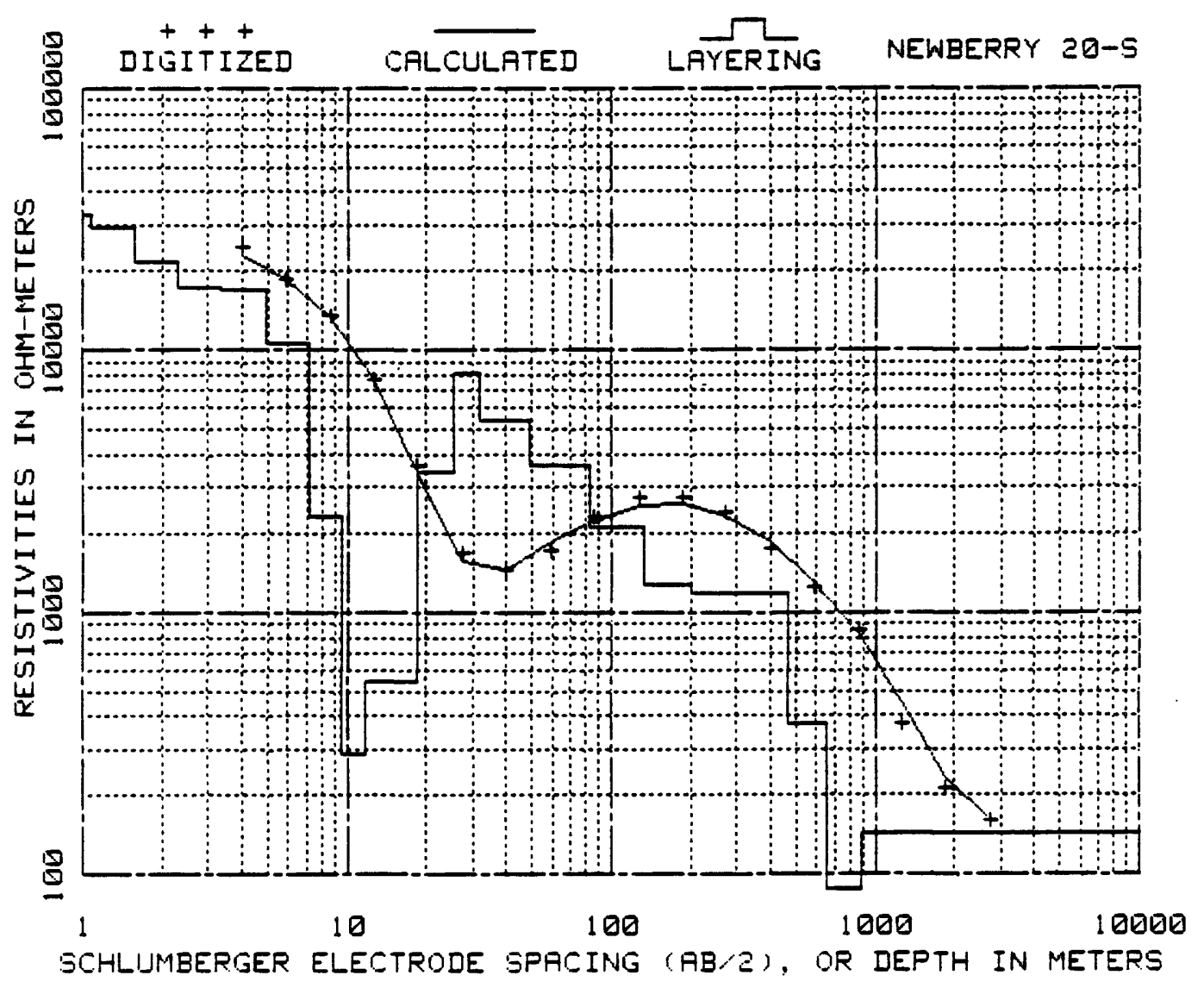

$\begin{array}{cc}\text { INTERPRETED } & \text { INTERPRETED } \\ \text { DEPTH } & \text { RESISTIVITY } \\ \text { IN METERS } & \text { IN OHM-METERS }\end{array}$

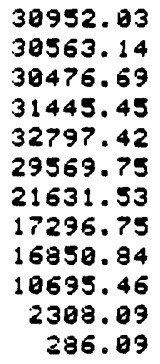

541.16

3419.90

8139.36

5415.32

3655.99

2097.05

1262.91

1177.43

1188.35

378.04

97.71

142. 97 


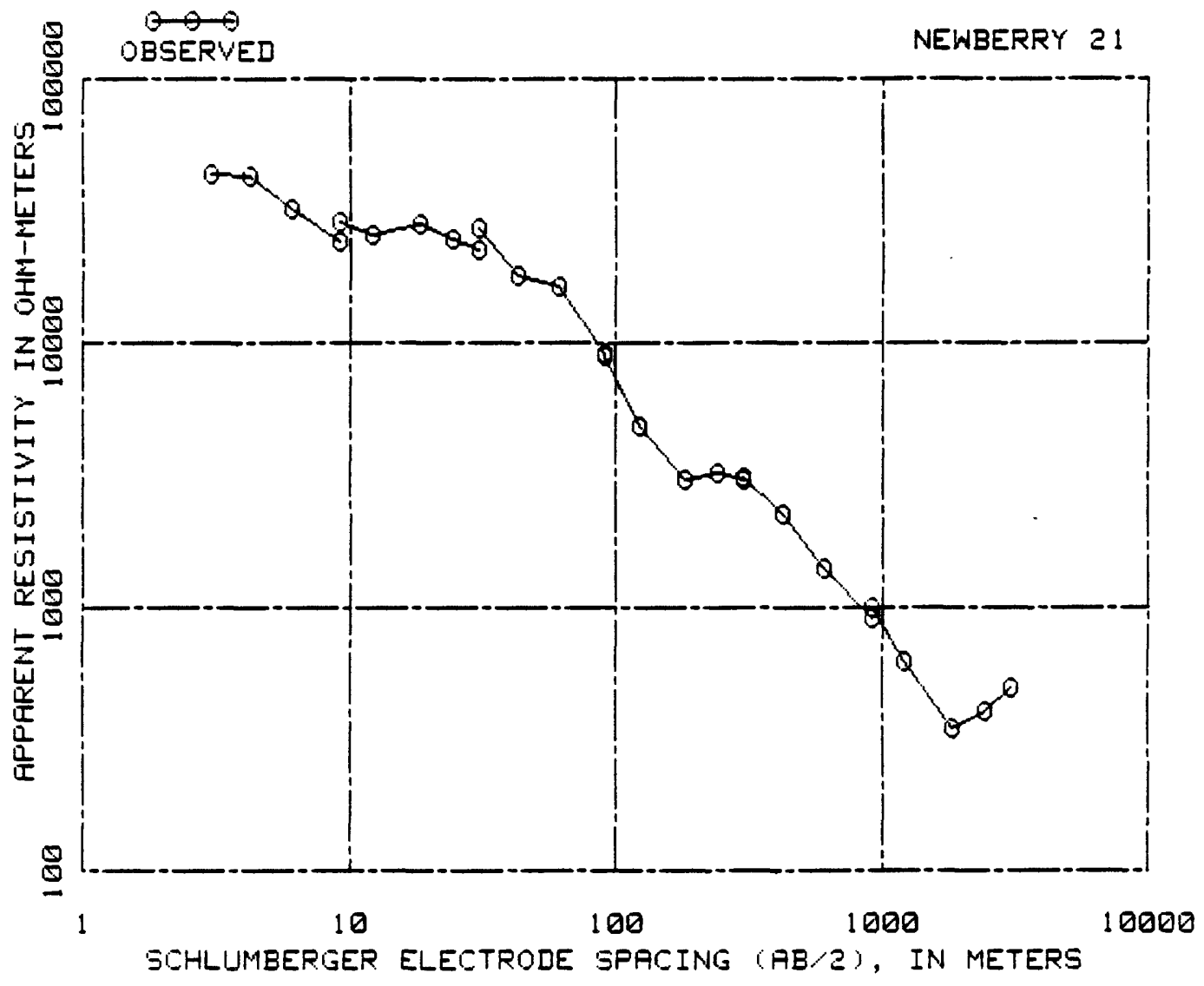

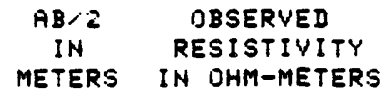

91.44
121.92
182.88
243.34
304.80
304.80
426.72
609.50
914.40
714.40
219.20
328.80
438.40
048.80

3900.00

4800.00

3000.00

3200.00

3000.00

3100.00

2250.00

1400.00

900.00

1000.00

530.00

350.00

400.00

500.00 


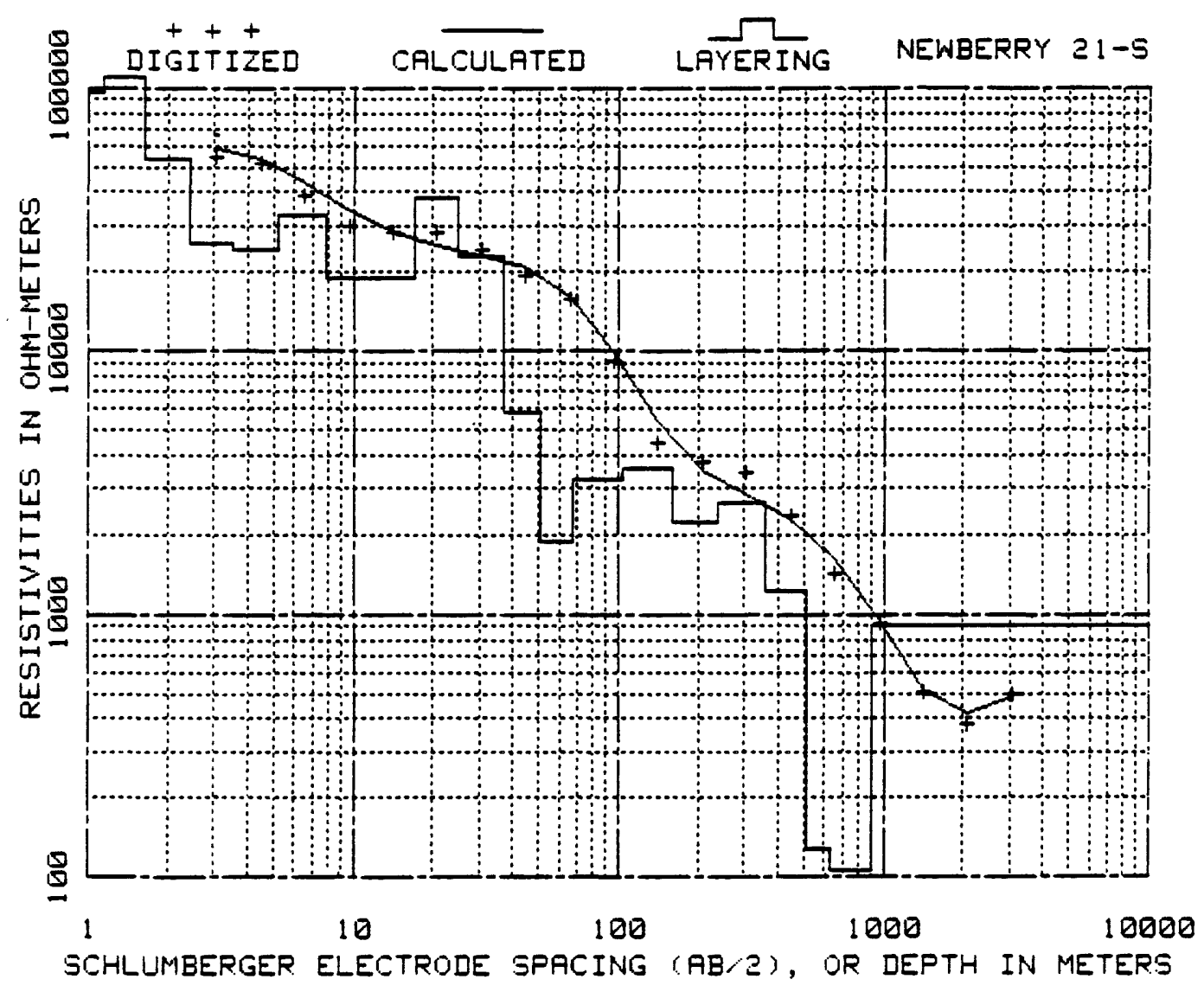

$\begin{array}{cr}\begin{array}{c}\text { INTERPRETED } \\ \text { DEPTH }\end{array} & \begin{array}{r}\text { INTERPRET } \\ \text { RESISTIVI } \\ \text { IN METERS } \\ \text { IN IHM-MET }\end{array} \\ & \\ .18 & +6682.01 \\ .26 & 49037.91 \\ .38 & 45011.97 \\ .56 & 40410.61 \\ .81 & 50636.00 \\ 1.13 & 96760.21 \\ 1.62 & 108840.93 \\ 2.44 & 53299.27 \\ 3.54 & 25657.00 \\ 5.33 & 24375.24 \\ 7.33 & 32990.56 \\ 11.49 & 19001.27\end{array}$

$\begin{array}{cc}\text { INTERPRETED } & \text { INTERPRETED } \\ \text { DEPTH } & \text { RESISTIVITY } \\ \text { IN METERS } & \text { IN OHM-METERS }\end{array}$

$\begin{array}{rr}16.99 & 19031.63 \\ 24.69 & 38429.86 \\ 36.79 & 22808.75 \\ 50.59 & 5859.88 \\ 67.40 & 1886.10 \\ 103.58 & 3235.07 \\ 158.86 & 3544.59 \\ 236.74 & 2232.90 \\ 356.48 & 2650.88 \\ 516.58 & 1219.00 \\ 632.63 & 127.73 \\ 894.36 & 105.99 \\ 1000893.36 & 908.14\end{array}$

